\title{
Prehistoric Plant Use at Beaver Creek Rock Shelter, Southwestern Montana, U.S.A.
}

\author{
Darla Dexter, Kathleen Martin, and Lauri Travis
}

\section{Research \\ Disclaimer}

The listing of plants in this article is not an endorsement of their use. Some of the plants are toxic. Before using any plants to treat a medical condition, medical advice should be obtained.

\begin{abstract}
The 2011 Carroll College Archaeological Field School conducted an exploratory excavation within the Beaver Creek Rock Shelter in southwestern Montana, U.S.A. The excavation exposed four cultural occupation layers dating to over 2,500 years ago. Pollen retrieved from the paleoenvironmental record included a wide variety of plants. Seven plant families were found in three of the occupation layers and in only one natural layer. This research reviewed the traditional Native American ethnobotanical uses of those seven plant families. They were used primarily for medicinal purposes. Although archaeologists have traditionally viewed botanical remains as evidence of prehistoric subsistence, this research demonstrates archaeologists' need to use caution in assuming plant remains in the archaeological record are predominately tied to subsistence.
\end{abstract}

\section{Introduction}

The 2011 Carroll College Archaeological Field School excavated the Beaver Creek Rock Shelter in southwestern Montana, U.S.A. The Beaver Creek Rock Shelter is a short-term campsite located on the west flank of the Big Belt Mountains in the Helena National Forest. Excavations revealed four cultural occupation layers dating to $1280 \pm 50$ BP (Beta-280431), 1730 \pm 40 BP (Beta-280432), 1980 \pm 50 BP (Beta-280433), and a layer dated between 1980 \pm 50 BP (Beta-280433) and 2470 \pm 30 BP (Beta-299721), ex- pressed in BP or years before present day, where "present day" is January 1950 (Travis et al. 2012).

Botanical remains were scarce within the Beaver Creek Rock Shelter sediments. The rock shelter strata did not contain any plant macrofossils. The only indication of plant utilization came from evidence within the pollen core. Pollen cores are typically used for paleoenvironmental information, not subsistence studies. We have concluded the pollen from the seven plant families represents botanical remains brought in to the rock shelter by prehistoric inhabitants as the pollen is found only in the three occupation layers. Fifteen of the 16 natural layers did not contain the seven pollen families. Plant macrofossils from archaeological sites may be disintegrated yet contain well-preserved pollen, indicating plant resources utilized (Faegri \& Iverson 1989:176). The pollen core from Beaver Creek Rock Shelter demonstrated that three occupation layers contained pollen from Apiaceae, Brassicaceae, Cyperaceae, Fabaceae, Geraniaceae, Lamiaceae, and Ranunculaceae, representing members of the umbel, mustard,

\section{Correspondence}

Darla Dexter, Lauri Travis, Carroll College, Department of Sociology and Anthropology, 1601 N. Benton Avenue, Helena, Montana 59625, U.S.A. Itravis@carroll.edu

Kathleen Martin, Carroll College, Corette Library, $1601 \mathrm{~N}$ Benton Avenue, Montana 59625, U.S.A.

Ethnobotany Research \& Applications 12:355-384 (2014)

Published: 07 September 2014 
sedge, legume, geranium, mint, and buttercup families, respectively (Travis et al. 2011). Pollen from these plant families were only found in the three occupation layers and a rapidly laid alluvial layer directly above Occupation 3. Nearly a meter of sediments was deposited above Occupation 3 in less than 250 years. It is probable that the pollen from the rapidly deposited sediments was attributed to the occupation layer below, especially as the pollen families were not found in any other natural layer in the entire two meters of deposits. As these plant families were only found in cultural layers and one incongruous layer, it is probable that the plants were brought in by the shortterm inhabitants of the rock shelter.

The Northern Plains have a rich history of ethnographic studies describing traditional Native American plant use. This paper will explore the traditional ethnographic Native American uses of the plant families found within the Beaver Creek Rock Shelter occupation layers excavated in 2011. Ethnographies of the Blackfeet, Cheyenne, Chippewa, Cree, Crow, Kutenai, Ojibwa, and Salish are included. The cultural affiliation of the Beaver Creek Rock Shelter is unknown due to the nomadic lifestyle of the listed tribes. However, it is known through historical records and oral histories that all of these tribes have periodically occupied the area in the last 3,000 years (Dusenberry \& Dusenberry Crow 1998, Ewers 1983, Greiser 1994, Grinnell 1972, Hannus 1994, Lowie \& Shapiro 1982, Schlesier 1994, Travis 1988).

\section{Environment}

The Beaver Creek Rock Shelter is located northeast of Helena, in southwestern Montana, U.S.A. The north-flowing Missouri River is bordered on the east by the Big Belt Mountains and the west by the Helena Valley. The rock shelter is located on the west flank of the Big Belt Mountains at the confluence of Beaver Creek and an unnamed intermittent drainage at $3840 \mathrm{ft}$ above sea-level. The dominant vegetation is a mixed Douglas fir - Ponderosa pine community. The Douglas fir - Ponderosa pine community is often separated by areas of mountain meadows or small, narrow riparian communities following the creeks. Beaver Creek runs northeast-southwest, and the unnamed intermittent drainage enters from the north creating a fairly large secondary terrace, about $6 \mathrm{~m}$ north-south by $10 \mathrm{~m}$ east-west. Beaver Creek is approximately $30 \mathrm{~m}$ south of the rock shelter and empties into the Missouri River to the southwest.
Changes in the paleoclimate undoubtedly affected the distribution of vegetation communities. Possible climate changes displayed by the Beaver Creek Rock Shelter pollen column have been discussed in a previous publication (Travis et al. 2012) and may be referred to for details.

\section{Cultural chronology of occupation}

Although excavations at the Beaver Creek Rock Shelter exposed limited cultural remains, four occupation layers were identified. Four radiocarbon dates were obtained from three distinct hearth features and one isolated charcoal crumble (Travis et al. 2012; Table 1).

The soil/pollen samples were collected from a single column isolated in the center of the north wall of test unit C3 (Travis et al. 2011; Figure 1). Samples were collected being extra careful not to mix strata. Two samples were collected from each stratum. A one-gallon bag was filled for gathering the granulometry data. A second smaller bag (about $1 / 2$ cup of soil) was collected for pollen samples. The pollen sample was collected from behind the granulometry sample as this soil was not exposed and less likely to be contaminated. Pollen samples were analyzed by the PaleoResearch Institute in Golden, Colorado.

Occupation 1, located 55-64 cm below surface (cmbs), dated to $1280 \pm 50 \mathrm{BP}$ (Beta-280431) and contained a single hearth feature, stone tools, flakes, and bone fragments from large mammals. A second hearth feature, $80 \mathrm{cmbs}$ in association with Occupation 2, had a radiocarbon date of $1730 \pm 40$ BP (Beta-280432). The level also had a single projectile point, flakes, and a wider variety of bone fragments from small game species. A third hearth feature, from Occupation 3, was located 169-180 cmbs and dated to $1980 \pm 50$ BP (Beta-280433). The occupation layer revealed only flakes and large mammal bone fragments. Occupation 4 was discovered just below Occupation 3 at $170-190 \mathrm{cmbs}$ and consisted of a very dark organic soil with heavy charcoal flecking and numerous bone chips throughout, but few other artifacts. The fourth occupational layer was dominated by large mammal bones with lesser amounts of small mammal bones. A radiocarbon date on a charcoal sample that was collected from 220-230 cmbs (about $50 \mathrm{~cm}$ below the base of Occupation 4) dated to $2470 \pm 30$ BP (Beta-299721). This date appears to be consistent with the site stratigraphic context; therefore, Occupation 4 likely dates between 1,980 BP and 2,470 BP (Travis et al. 2012).

Table 1. Radiocarbon dates for Beaver Creek Rock Shelter, southwestern Montana, U.S.A.

\begin{tabular}{|l|c|c|c|c|c|}
\hline Sample & Measured Age & Technique & Conventional Age & Stratum & 2 $\boldsymbol{\sigma}$ Calibrated Age \\
\hline Beta-280431 & $1290 \pm 50 \mathrm{BP}$ & standard & $1280 \pm 50 \mathrm{BP}$ & 15 & A.D. $650-880$ \\
\hline Beta-280432 & $1740 \pm 40 \mathrm{BP}$ & standard & $1730 \pm 40 \mathrm{BP}$ & 13 & A.D. $230-410$ \\
\hline Beta-280433 & $1940 \pm 50 \mathrm{BP}$ & standard & $1980 \pm 50 \mathrm{BP}$ & 6 & B.C. $90-$ A.D. 120 \\
\hline Beta-299721 & $2410 \pm 30 \mathrm{BP}$ & AMS & $2470 \pm 30 \mathrm{BP}$ & 1 & B.C. $760-410$ \\
\hline
\end{tabular}


Dexter et al. - Prehistoric Plant Use at Beaver Creek Rock Shelter,

Southwestern Montana, U.S.A.

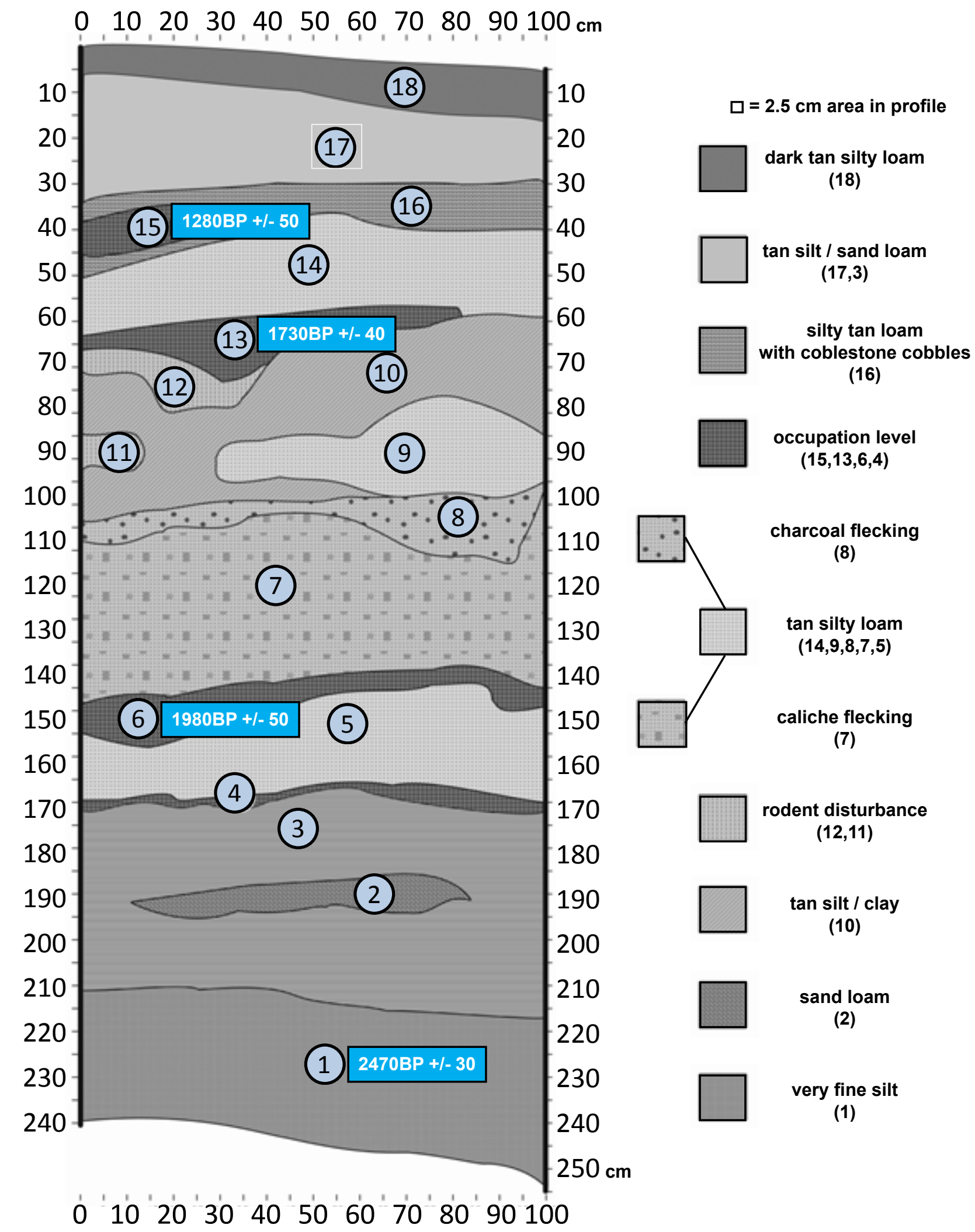

Figure 1. Beaver Creek Rock Shelter Site 24LC1993/2186, southwestern Montana, U.S.A. Profile North Wall Unit C3 (after Travis et al. 2011). 


\section{Plant families}

The prairies, valleys, wetlands, and mountain forests of Montana support a wide diversity of plant life with 128 plant families and over 2,082 native species (Lesica 2012). Seven families were exclusively identified from pollen in the three occupation layers (Travis et al. 2011, Travis et al. 2012; Figure 2). Five of the seven families iden-

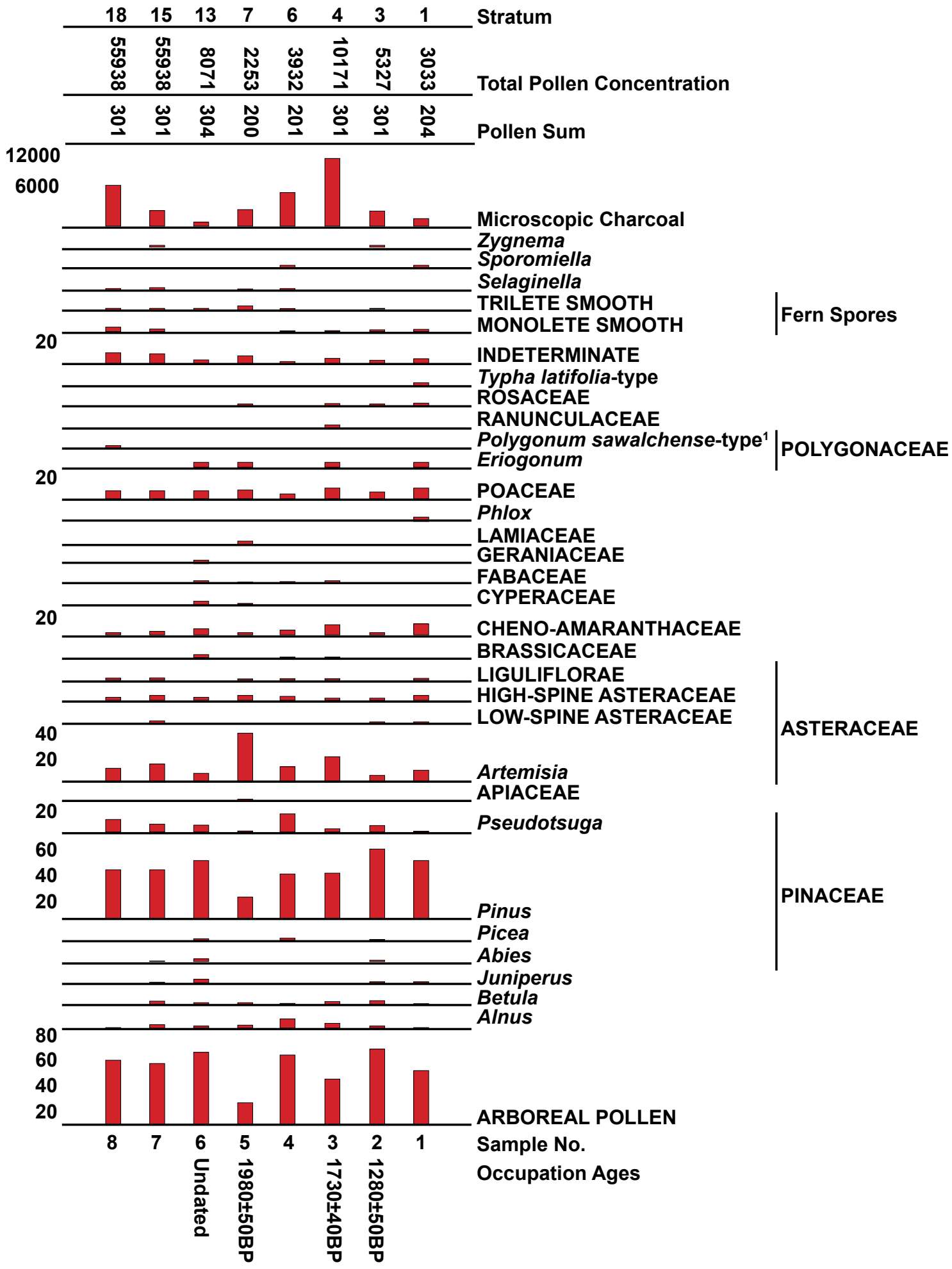

Figure 2. Pollen profile, Beaver Creek Rock Shelter, southwestern Montana, U.S.A. (after Cummings \& Yost 2011). 1. Polygonum sawalchense is a synonym of $P$. douglasii subsp. johnstonii (Munz) J.C.Hickman 


\section{Southwestern Montana, U.S.A.}

tified in this study are ranked in the top ten largest plant families in Montana. The families from the pollen samples consist of Cyperaceae (with 163 native species ranks second), Brassicaceae (with 99 species ranks fourth), Fabaceae (with 98 species ranks fifth), Ranunculaceae (with 62 species ranks seventh), and Apiaceae (with 49 species ranks tenth) (Lesica 2012). The two smaller families, Lamiaceae and Geraniaceae, have 38 species and 10 species, respectively.

\section{Methods}

Due to the large number of species in these families and the inability to identify the herbal pollen to genus or species, we can only speculate about which exact plant species were in the occupational layers. The final list of plant species was derived from a two-step process. First, all Montana native plants from the seven pollen families were identified (Mincemoyer 2012). Second, each species was investigated for ethnographic use by the Native American tribes that are known to have occupied the area. If a species was not found in the regional ethnographic literature, it was removed from the list. The results of the above comparisons are detailed below.

\section{Results}

\section{Biogeography}

Lesica (2012) classified Montana vegetation life zones into five types: plains, valleys, montane, subalpine, and alpine. All life zones with the exception of the plains occur in the mountainous regions of Montana. The plains region refers to the Great Plains of eastern Montana. Elevations range from $550 \mathrm{~m}(1,800 \mathrm{ft})$ in the plains to above 3,000 $\mathrm{m}(9,500 \mathrm{ft})$ in the alpine zone. The Beaver Creek Rock Shelter occurs at the lower elevation of the montane zone which encompasses the lowest and warmest portions of mountainous terrain. The majority of the ethnobotanical species listed are found in low to mid-elevations of the plains, meadows, and montane regions of Montana, with fewer species occurring in the higher subalpine and alpine zones (Table 2).

Table 2. Geographic location of selected plant species in Montana, U.S.A. Montana vegetation life zones: (PI) Plains (548-914 m), (Va)Valleys (NW 610-915 m to over $1525 \mathrm{~m}$ in the SW \& S-central), (Mo) Montane (NW 760-1680 m and 1370-2130 m east of the Contenental Divide), (Su) Subalpine (NW 1675-2135 m and 1980-2900 in the SW and S-central mountain ranges), and (Al) Alpine (NW 1980-2130 m and above $3000 \mathrm{~m}$ in SW). ( $\Delta$ ) Location, or (- $\Delta$ ) Lower altitude location. Species distribution only to the closest proximity of the study site: $(A)$ in Lewis \& Clark County, (B) in adjoining counties, (C) outside of Lewis and Clark and adjoining counties.

\begin{tabular}{|c|c|c|c|c|c|c|c|c|c|}
\hline \multicolumn{2}{|l|}{ Plant Names } & \multicolumn{5}{|c|}{ Montana vegetation life zones } & \multicolumn{3}{|c|}{$\begin{array}{c}\text { Species } \\
\text { distribution }\end{array}$} \\
\hline Scientific & Common & $\mathbf{P I}$ & $\mathbf{V a}$ & Mo & Su & Al & A & B & C \\
\hline \multicolumn{10}{|l|}{ Apiaceae } \\
\hline Angelica dawsonii S.Watson & Dawson's angelica & & & $\Delta$ & $\Delta$ & & & & $\Delta$ \\
\hline $\begin{array}{l}\text { Cicuta douglasii (DC.) } \\
\text { J.M.Coult. \& Rose }\end{array}$ & Western water-hemlock & & $\Delta$ & $\Delta$ & & & & $\Delta$ & \\
\hline Cicuta maculata L. & Spotted water-hemlock & $\Delta$ & $\Delta$ & & & & $\Delta$ & & \\
\hline Ligusticum canbyi J.M.Coult. \& Rose & Canby's wild lovage & & & $\Delta$ & & & $\Delta$ & & \\
\hline $\begin{array}{l}\text { Lomatium ambiguum (Nutt.) } \\
\text { J.M.Coult. \& Rose }\end{array}$ & Wyeth biscuitroot & & $\Delta$ & $\Delta$ & & & $\Delta$ & & \\
\hline $\begin{array}{l}\text { Lomatium cous (S.Watson) } \\
\text { J.M.Coult. \& Rose }\end{array}$ & Cous biscuitroot & $\Delta$ & $\Delta$ & $\Delta$ & $\Delta$ & $\Delta$ & $\Delta$ & & \\
\hline $\begin{array}{l}\text { Lomatium macrocarpum (Hook. } \\
\text { \& Arn.) J.M.Coult. \& Rose }\end{array}$ & Large-fruit desert-parsley & $\Delta$ & $\Delta$ & $\Delta$ & & & $\Delta$ & & \\
\hline Lomatium orientale J.M.Coult. \& Rose & Oriental desert-parsley & $\Delta$ & $\Delta$ & & & & & & $\Delta$ \\
\hline $\begin{array}{l}\text { Lomatium triternatum (Pursh) } \\
\text { J.M.Coult. \& Rose }\end{array}$ & Nineleaf biscuitroot & & $\Delta$ & $\Delta$ & & & $\Delta$ & & \\
\hline Musineon divaricatum (Pursh) Nutt. & Wild parsley & $\Delta$ & $\Delta$ & & & & $\Delta$ & & \\
\hline Osmorhiza chilensis Hook. \& Arn. & Chilean sweet-cicely & & $\Delta$ & $\Delta$ & $-\Delta$ & & $\Delta$ & & \\
\hline Osmorhiza longistylis (Torr.) DC. & Smoother sweet-cicely & $\Delta$ & $\Delta$ & & & & & $\Delta$ & \\
\hline Osmorhiza occidentalis (Nutt.) Torr. & Western sweet-cicely & & $\Delta$ & $\Delta$ & $\Delta$ & & $\Delta$ & & \\
\hline Perideridia montana (Blank.) Dorn & Gairdner's yampah & & $\Delta$ & $\Delta$ & $-\Delta$ & & $\Delta$ & & \\
\hline
\end{tabular}




\begin{tabular}{|c|c|c|c|c|c|c|c|c|c|}
\hline \multicolumn{2}{|l|}{ Plant Names } & \multicolumn{5}{|c|}{ Montana vegetation life zones } & \multicolumn{3}{|c|}{$\begin{array}{c}\text { Species } \\
\text { distribution }\end{array}$} \\
\hline Scientific & Common & PI & $\mathrm{Va}$ & Mo & Su & Al & A & B & C \\
\hline Sanicula marilandica L. & Maryland black snakeroot & $\Delta$ & $\Delta$ & $-\Delta$ & & & & $\Delta$ & \\
\hline Sium suave Walter & Hemlock water-parsnip & $\Delta$ & $\Delta$ & $\Delta$ & & & & $\Delta$ & \\
\hline \multicolumn{10}{|l|}{ Brassicaceae } \\
\hline Draba incerta Payson & Yellowstone whitlow-grass & & & & & $\Delta$ & $\Delta$ & & \\
\hline Erysimum cheiranthoides $\mathrm{L}$. & Wormseed wallflower & $\Delta$ & $\Delta$ & $\Delta$ & & & $\Delta$ & & \\
\hline Physaria didymocarpa (Hook.) A.Gray & Common twinpod & $\Delta$ & $\Delta$ & $\Delta$ & & & $\Delta$ & & \\
\hline Turritis glabra L. & Tower-mustard & $\Delta$ & $\Delta$ & $\Delta$ & & & $\Delta$ & & \\
\hline \multicolumn{10}{|l|}{ Cyperaceae } \\
\hline $\begin{array}{l}\text { Amphiscirpus nevadensis } \\
\text { (S.Watson) Oteng-Yeb. }\end{array}$ & Nevada bulrush & $\Delta$ & $\Delta$ & & & & & $\Delta$ & \\
\hline Carex nebraskensis Dewey & Nebraska sedge & $\Delta$ & $\Delta$ & $\Delta$ & & & $\Delta$ & & \\
\hline $\begin{array}{l}\text { Eriophorum callitrix } \\
\text { Cham. ex C.A.Mey. }\end{array}$ & Sheathed cotton-grass & & & & & $\Delta$ & & & $\Delta$ \\
\hline $\begin{array}{l}\text { Schoenoplectus acutus (Muhl. } \\
\text { ex Bigelow) Á.Löve \& D.Löve }\end{array}$ & Hardstem bulrush & $\Delta$ & $\Delta$ & $\Delta$ & & & $\Delta$ & & \\
\hline $\begin{array}{l}\text { Schoenoplectus americanus } \\
\text { (Pers.) Volkart }\end{array}$ & Three-square bulrush & & $\Delta$ & & & & & & $\Delta$ \\
\hline Scirpus cyperinus (L.) Kunth & Woolgrass & & $\Delta$ & & & & & & $\Delta$ \\
\hline \multicolumn{10}{|l|}{ Fabaceae } \\
\hline Amorpha canescens Pursh & Lead plant & $\Delta$ & & & & & & & $\Delta$ \\
\hline $\begin{array}{l}\text { Astragalus americanus } \\
\text { (Hook.) M.E.Jones }\end{array}$ & American milkvetch & & $\Delta$ & $\Delta$ & $-\Delta$ & & & $\Delta$ & \\
\hline Astragalus canadensis L. & Canadian milkvetch & $\Delta$ & $\Delta$ & $\Delta$ & & & $\Delta$ & & \\
\hline Astragalus crassicarpus Nutt. & Groundplum milkvetch & $\Delta$ & $\Delta$ & & & & $\Delta$ & & \\
\hline Dalea candida Willd. & White prairie clover & $\Delta$ & & & & & $\Delta$ & & \\
\hline Dalea purpurea Vent. & Purple prairie clover & $\Delta$ & $\Delta$ & & & & $\Delta$ & & \\
\hline Glycyrrhiza lepidota Pursh & Wild licorice & $\Delta$ & $\Delta$ & & & & $\Delta$ & & \\
\hline Lupinus polyphyllus Lindl. & Bigleaf lupine & $\Delta$ & $\Delta$ & $\Delta$ & $\Delta$ & & & $\Delta$ & \\
\hline Oxytropis lagopus Nutt. & Hare's-foot locoweed & & $\Delta$ & $\Delta$ & & & $\Delta$ & & \\
\hline Oxytropis sericea Torr. \& A.Gray & White locoweed & $\Delta$ & $\Delta$ & $\Delta$ & $\Delta$ & $\Delta$ & $\Delta$ & & \\
\hline $\begin{array}{l}\text { Pediomelum argophyllum } \\
\text { (Pursh) J.W.Grimes }\end{array}$ & Silvery scurfpea & $\Delta$ & $\Delta$ & & & & & & $\Delta$ \\
\hline $\begin{array}{l}\text { Pediomelum esculentum } \\
\text { (Pursh) Rydb. }\end{array}$ & Pomme de prairie & $\Delta$ & & & & & $\Delta$ & & \\
\hline Psoralea hypogaea Torr. \& A.Gray & Little indian breadroot & $\Delta$ & & & & & & $\Delta$ & \\
\hline Psoralea lanceolata Pursh & Lance-leaf scurfpea & $\Delta$ & $\Delta$ & $\Delta$ & & & & & $\Delta$ \\
\hline $\begin{array}{l}\text { Thermopsis rhombifolia } \\
\text { (Pursh) Richardson }\end{array}$ & Roundleaf thermopsis & $\Delta$ & $\Delta$ & & & & $\Delta$ & & \\
\hline Vicia americana Willd. & American purple vetch & $\Delta$ & $\Delta$ & $\Delta$ & & & $\Delta$ & & \\
\hline \multicolumn{10}{|l|}{ Geraniaceae } \\
\hline $\begin{array}{l}\text { Geranium richardsonii } \\
\text { Fisch. \& Trautv. }\end{array}$ & Richardson's geranium & & $\Delta$ & $\Delta$ & & & $\Delta$ & & \\
\hline
\end{tabular}


Dexter et al. - Prehistoric Plant Use at Beaver Creek Rock Shelter, Southwestern Montana, U.S.A.

\begin{tabular}{|l|l|l|l|l|l|l|l|l|l|}
\hline Plant Names & \multicolumn{3}{l|}{ Montana vegetation life zones } & \multicolumn{2}{|l|}{$\begin{array}{l}\text { Species } \\
\text { distribution }\end{array}$} \\
\hline Scientific & Common & PI & Va & Mo & Su & Al & A & B & C \\
\hline $\begin{array}{l}\text { Geranium viscosissimum } \\
\text { Fisch. \& C.A.Mey. }\end{array}$ & Sticky geranium & & $\Delta$ & $\Delta$ & $-\Delta$ & & $\Delta$ & & \\
\hline Lamiaceae & Mint & & & & & & & & \\
\hline Agastache foeniculum (Pursh) Kuntze & Lavender hyssop & $\Delta$ & & & & & & & $\Delta$ \\
\hline Lycopus asper Greene & Rough bugleweed & $\Delta$ & $\Delta$ & & & & & $\Delta$ & \\
\hline Mentha arvensis L. & Wild mint & $\Delta$ & $\Delta$ & $\Delta$ & & & $\Delta$ & & \\
\hline Monarda fistulosa L. & Beebalm & $\Delta$ & $\Delta$ & $\Delta$ & & & $\Delta$ & & \\
\hline Prunella vulgaris L. & Self-heal & & $\Delta$ & $\Delta$ & & & $\Delta$ & & \\
\hline Scutellaria galericulata L. & Hooded skullcap & $\Delta$ & $\Delta$ & $\Delta$ & & & $\Delta$ & & \\
\hline Stachys palustris L. & Marsh hedge-nettle & $\Delta$ & $\Delta$ & $\Delta$ & & & $\Delta$ & & \\
\hline Ranunculaceae & & & & & & \\
\hline Actaea rubra (Aiton) Willd. & Red baneberry & $\Delta$ & $\Delta$ & $\Delta$ & $-\Delta$ & & $\Delta$ & & \\
\hline Anemone canadensis L. & Canada anemone & $\Delta$ & & & & & & & $\Delta$ \\
\hline Anemone cylindrica A.Gray & Long-fruited anemone & $\Delta$ & $\Delta$ & $\Delta$ & & & $\Delta$ & & \\
\hline Anemone multifida Poir. & Pacific anemone & $\Delta$ & $\Delta$ & $\Delta$ & $\Delta$ & $\Delta$ & $\Delta$ & & \\
\hline Clematis hirsutissima Pursh & Sugarbowls & & $\Delta$ & $\Delta$ & $\Delta$ & & $\Delta$ & & \\
\hline Clematis ligusticifolia Nutt. & Western virgin's-bower & $\Delta$ & $\Delta$ & $\Delta$ & & & $\Delta$ & & \\
\hline Delphinium bicolor Nutt. & Little larkspur & $\Delta$ & $\Delta$ & $\Delta$ & $\Delta$ & $\Delta$ & $\Delta$ & & \\
\hline Ranunculus pensylvanicus L.f. & Bristly crowfoot & $\Delta$ & $\Delta$ & & & & & & $\Delta$ \\
\hline $\begin{array}{l}\text { Thalictrum dasycarpum Fisch., } \\
\text { C.A.Mey. \& Avé-Lall. }\end{array}$ & Purple meadowrue & $\Delta$ & $\Delta$ & $\Delta$ & & & & $\Delta$ & \\
\hline Thalictrum occidentale A.Gray & Western meadowrue & & & $\Delta$ & $\Delta$ & & $\Delta$ & & \\
\hline
\end{tabular}

Fifty of the 61 ethnobotanical species listed currently occur in Lewis and Clark County or the adjoining counties of Flathead, Teton, Cascade, Meagher, Broadwater, Jefferson, and Powell. The remaining 11 species that were found outside of Lewis and Clark County and adjacent counties consist of two species in Apiaceae, three in Fabaceae, one in Lamiaceae, two in Ranunculaceae, and three in Cyperaceae. The following is a list of those 11 species along with their occurrence in Montana: Angelica dawsonii S.Watson, is endemic to Idaho, northwest Montana, and adjacent British Columbia, Alberta, Canada; Lomatium orientale J.M.Coult. \& Rose is found in southeast Montana; Amorpha canescens Pursh, a Great Plains species was collected only once in southeast Montana about 70 years ago; Psoralea argophylla Pursh is found in the eastern half of Montana; Psoralea lanceolata Pursh is found mostly in the eastern half of Montana and in one county in southwestern Montana; Agastache foeniculum (Pursh) Kuntze is known from only two counties in eastern Montana; Anemone canadensis L. is found in northeast Montana; Ranunculus pensylvanicus L.f. is found in western and south-central Montana; Eriophorum callitrix Cham. ex C.A.Mey. is found only in south-central Mon- tana; Schoenoplectus americanus (Pers.) Volkart is found in two counties in southwestern Montana; and Scirpus cyperinus (L.) Kunth is found in only one county in northwest Montana (Lesica 2012). It is important to note that these species may occur in the surrounding area but have not been documented or that they may have existed in the area historically but conditions may have changed in climate or land-use that precludes their existence in modern times.

The presence of plant resources from distant areas was expected, as prehistoric trade systems have been well documented (Baugh \& Ericson 1994, Boyd 1998, Carlson 1994, Cooper 2008, Galm 1994, Vehik \& Baugh 1994, Wood 1974) and Northern Plains tribes were known to travel substantial distances in their seasonal rounds (Duke \& Wilson 1994, Frison 1991, 1998, Greiser 1994, Reeves 1970). The Beaver Creek Rock Shelter excavation yielded additional evidence of long-distance trade/travel in the form of obsidian and Sauger fish remains. Obsidian from the Beaver Creek Rock Shelter was sourced to Bear Gulch located in southern Idaho (Travis et al. 2011). The excavation also revealed Sauger fish bones, which are 
currently and historically found only in the middle and lower Missouri River, not the upper Missouri where Beaver Creek is located (Travis et al. 2011).

\section{Northern Plains ethnographic plant use}

Numerous sources in literature document traditional Native American plant use. The following section contains the most commonly recorded species from the 7 families that were known to be utilized by Montana Native Americans. Each table contains the tribe or tribes, the plant part, and how the plant was prepared.

\section{Food, beverages, and food-related ethnographic uses}

Great Plains Native Americans utilized 25 different plant species for subsistence (Table 3). Subsistence uses were dominated by species and particularly roots in the Faba-

Table 3. Plants implied from the archaeological record at Beaver Creek Rock Shelter, southwestern Montana, U.S.A. with ethnographic uses as foods, beverages, and food-related purposes. Information sources: (B) Blankinship 1905, (C) Clavell 1997, (D) Densmore 1927, (G) Grinnell 1972, (H1) Hart 1981, (H2) Hart 1992, (J) Johnston 1970, (K1) Kindscher 1987, (K2) Kindscher 1992, (M) Moerman 1998, (T1) Taylor 1989, and (T2) Teit 1928.

\begin{tabular}{|c|c|c|c|c|c|c|}
\hline \multicolumn{2}{|l|}{ Plant names } & \multirow{2}{*}{$\begin{array}{l}\text { Cultural } \\
\text { source }\end{array}$} & \multicolumn{3}{|c|}{ Plant information } & \multirow[t]{2}{*}{ Sources } \\
\hline Scientific & Common & & Uses & Parts used & Preparation & \\
\hline \multicolumn{7}{|l|}{ Apiaceae } \\
\hline $\begin{array}{l}\text { Lomatium } \\
\text { ambiguum (Nutt.) } \\
\text { J.M.Coult. \& Rose }\end{array}$ & $\begin{array}{l}\text { Wyeth } \\
\text { biscuitroot }\end{array}$ & $\begin{array}{l}\text { Native } \\
\text { American }\end{array}$ & food & root & reduced to flour & $\mathrm{B}, \mathrm{M}$ \\
\hline $\begin{array}{l}\text { Lomatium cous } \\
\text { (S. Watson) } \\
\text { J.M.Coult. \& Rose }\end{array}$ & $\begin{array}{l}\text { Cous } \\
\text { biscuitroot }\end{array}$ & MT Indian & food & root & $\begin{array}{l}\text { whole roots dried, } \\
\text { pulverized for } \\
\text { porridge, cakes or } \\
\text { soup, peeled and } \\
\text { eaten raw or boiled }\end{array}$ & $\mathrm{B}, \mathrm{H} 2, \mathrm{M}$ \\
\hline $\begin{array}{l}\text { Lomatium } \\
\text { macrocarpum } \\
\text { (Hook. \& Arn.) } \\
\text { J.M.Coult. \& Rose }\end{array}$ & $\begin{array}{l}\text { Large-fruit } \\
\text { desert- } \\
\text { parsley }\end{array}$ & Salish & food & root & eaten dried or raw & $\mathrm{K} 1, \mathrm{M}$ \\
\hline \multirow{4}{*}{$\begin{array}{l}\text { Lomatium } \\
\text { triternatum (Pursh) } \\
\text { J.M.Coult. \& Rose }\end{array}$} & \multirow[t]{4}{*}{$\begin{array}{l}\text { Nineleaf } \\
\text { biscuitroot }\end{array}$} & \multirow[t]{3}{*}{ Blackfeet } & \multirow[t]{2}{*}{ food } & flower & $\begin{array}{l}\text { used to make } \\
\text { pemmican }\end{array}$ & $M$ \\
\hline & & & & root & eaten raw or roasted & $M$ \\
\hline & & & preservative & fruit & $\begin{array}{l}\text { to keep the hide } \\
\text { of an animal } \\
\text { from smelling }\end{array}$ & $M$ \\
\hline & & MT Indian & food & root & $\begin{array}{l}\text { reduced to flour } \\
\text { or eaten raw, } \\
\text { roasted, or baked }\end{array}$ & $\mathrm{B}, \mathrm{M}$ \\
\hline $\begin{array}{l}\text { Musineon } \\
\text { divaricatum (Pursh) } \\
\text { Nutt. }\end{array}$ & Wild parsley & Blackfeet & food & root & eaten raw & $M$ \\
\hline $\begin{array}{l}\text { Osmorhiza chilensis } \\
\text { Hook. \& Arn. }\end{array}$ & $\begin{array}{l}\text { Western } \\
\text { sweet-cicely }\end{array}$ & Blackfeet & food & root & $\begin{array}{l}\text { chewed and } \\
\text { eaten as candy }\end{array}$ & $M$ \\
\hline \multirow{5}{*}{$\begin{array}{l}\text { Perideridia montana } \\
\text { (Blank.) Dorn }\end{array}$} & \multirow{5}{*}{$\begin{array}{l}\text { Gairdner's } \\
\text { Yampah }\end{array}$} & \multirow[t]{2}{*}{ Blackfeet } & \multirow[t]{2}{*}{ food } & \multirow[t]{2}{*}{ root } & eaten raw as a snack & $M$ \\
\hline & & & & & $\begin{array}{l}\text { stored for soup } \\
\text { or a staple }\end{array}$ & $M$ \\
\hline & & Cheyenne & food & root & $\begin{array}{l}\text { scraped, dried, and } \\
\text { stored or pulverized } \\
\text { and eaten as mush }\end{array}$ & $\mathrm{H} 1, \mathrm{M}$ \\
\hline & & Salish & food & root & $\begin{array}{l}\text { smashed, formed } \\
\text { into bread or cakes, } \\
\text { and sun dried }\end{array}$ & $M$ \\
\hline & & MT Indian & food & root & eaten raw or boiled & $M$ \\
\hline
\end{tabular}


Dexter et al. - Prehistoric Plant Use at Beaver Creek Rock Shelter,

Southwestern Montana, U.S.A.

\begin{tabular}{|c|c|c|c|c|c|c|}
\hline \multicolumn{2}{|l|}{ Plant names } & \multirow{2}{*}{$\begin{array}{l}\text { Cultural } \\
\text { source }\end{array}$} & \multicolumn{3}{|c|}{ Plant information } & \multirow[t]{2}{*}{ Sources } \\
\hline Scientific & Common & & Uses & Parts used & Preparation & \\
\hline \multirow[t]{2}{*}{ Sium suave Walter } & \multirow{2}{*}{$\begin{array}{l}\text { Hemlock } \\
\text { water- } \\
\text { parsnip }\end{array}$} & Cree & food & root & $\begin{array}{l}\text { eaten roasted, } \\
\text { raw, or fried }\end{array}$ & C, M \\
\hline & & MT Indian & food & herbage & $\begin{array}{l}\text { eaten as a relish due } \\
\text { to its aromatic flavor }\end{array}$ & $\mathrm{B}, \mathrm{M}$ \\
\hline \multicolumn{7}{|l|}{ Brassicaceae } \\
\hline Turritis glabra L. & $\begin{array}{l}\text { Tower- } \\
\text { mustard }\end{array}$ & Cheyenne & beverage & unknown & infusion & G, M \\
\hline \multicolumn{7}{|l|}{ Cyperaceae } \\
\hline $\begin{array}{l}\text { Amphiscirpus } \\
\text { nevadensis } \\
\text { (S.Watson) } \\
\text { Oteng-Yeb. }\end{array}$ & $\begin{array}{l}\text { Nevada } \\
\text { bulrush }\end{array}$ & Cheyenne & food & root & $\begin{array}{l}\text { peeled and } \\
\text { eaten raw }\end{array}$ & $\mathrm{G}, \mathrm{M}$ \\
\hline \multirow{5}{*}{$\begin{array}{l}\text { Schoenoplectus } \\
\text { acutus (Muhl. ex } \\
\text { Bigelow) Á.Löve } \\
\text { \& D.Löve }\end{array}$} & \multirow{5}{*}{$\begin{array}{l}\text { Hardstem } \\
\text { bulrush }\end{array}$} & Cree & food & stem & eaten raw & $\mathrm{J}, \mathrm{M}$ \\
\hline & & Blackfeet & food & root & eaten raw or cooked & $\mathrm{J}, \mathrm{M}$ \\
\hline & & \multirow[t]{2}{*}{ MT Indian } & \multirow[t]{2}{*}{ food } & \multirow[t]{2}{*}{ root } & $\begin{array}{l}\text { made into syrup, } \\
\text { sauce, or relish }\end{array}$ & $\mathrm{B}, \mathrm{M}$ \\
\hline & & & & & $\begin{array}{l}\text { eaten raw or made } \\
\text { into flour for bread }\end{array}$ & $\mathrm{B}, \mathrm{M}$ \\
\hline & & Cheyenne & food & stem & eaten raw & $\mathrm{H} 1, \mathrm{M}$ \\
\hline \multicolumn{7}{|l|}{ Fabaceae } \\
\hline $\begin{array}{l}\text { Astragalus } \\
\text { canadensis L. }\end{array}$ & $\begin{array}{l}\text { Canadian } \\
\text { milkvetch }\end{array}$ & Blackfeet & food & root & $\begin{array}{l}\text { eaten raw or } \\
\text { boiled in blood } \\
\text { to make broth }\end{array}$ & $\mathrm{K} 1, \mathrm{M}$ \\
\hline \multirow{3}{*}{$\begin{array}{l}\text { Astragalus } \\
\text { crassicarpus Nutt. }\end{array}$} & \multirow{3}{*}{$\begin{array}{l}\text { Groundplum } \\
\text { milkvetch }\end{array}$} & Chippewa & tonic & root & decoction & $\mathrm{D}, \mathrm{K} 1, \mathrm{M}$ \\
\hline & & \multirow[t]{2}{*}{ MT Indian } & \multirow[t]{2}{*}{ food } & fruit & eaten raw as a snack & $\mathrm{B}, \mathrm{M}$ \\
\hline & & & & pods & $\begin{array}{l}\text { eaten raw, cooked, } \\
\text { or pickled }\end{array}$ & $\mathrm{B}, \mathrm{M}$ \\
\hline \multirow{3}{*}{$\begin{array}{l}\text { Dalea candida } \\
\text { Willd. }\end{array}$} & \multirow{3}{*}{$\begin{array}{l}\text { White prairie } \\
\text { clover }\end{array}$} & Blackfeet & food & root & eaten raw & $\mathrm{K} 1, \mathrm{~K} 2$ \\
\hline & & \multirow[t]{2}{*}{ MT Indian } & beverage & leaves & unspecified & $\mathrm{K} 1, \mathrm{~K} 2$ \\
\hline & & & food & root & unspecified & $\mathrm{K} 1, \mathrm{~K} 2$ \\
\hline \multirow{2}{*}{$\begin{array}{l}\text { Dalea purpurea } \\
\text { Vent. }\end{array}$} & \multirow{2}{*}{$\begin{array}{l}\text { Purple } \\
\text { prairie clover }\end{array}$} & \multirow[t]{2}{*}{ MT Indian } & food & root & unspecified & $\mathrm{K} 2, \mathrm{M}$ \\
\hline & & & beverage & leaves & unspecified & $\mathrm{K} 2, \mathrm{M}$ \\
\hline \multirow[t]{3}{*}{$\begin{array}{l}\text { Glycyrrhiza lepidota } \\
\text { Pursh }\end{array}$} & \multirow[t]{3}{*}{ Wild licorice } & Cheyenne & food & shoots & eaten raw & $\begin{array}{l}\mathrm{H} 2, \\
\mathrm{~K} 1, \mathrm{M} \\
\end{array}$ \\
\hline & & \multirow[t]{2}{*}{ MT Indian } & tonic & \multirow[t]{2}{*}{ root } & infusion & $\mathrm{H} 2, \mathrm{M}$ \\
\hline & & & food & & unspecified & $\mathrm{H} 2, \mathrm{M}$ \\
\hline $\begin{array}{l}\text { Lupinus polyphyllus } \\
\text { Lindl. }\end{array}$ & $\begin{array}{l}\text { Bigleaf } \\
\text { lupine } \\
\end{array}$ & Salish & tonic & unknown & decoction & $\mathrm{M}, \mathrm{T} 2$ \\
\hline \multirow[t]{2}{*}{$\begin{array}{l}\text { Pediomelum } \\
\text { esculentum } \\
\text { (Pursh) Rydb. }\end{array}$} & \multirow[t]{2}{*}{$\begin{array}{l}\text { Pomme de } \\
\text { prairie }\end{array}$} & Blackfeet & food & root & $\begin{array}{l}\text { eaten raw or } \\
\text { peeled and dried }\end{array}$ & $\begin{array}{l}\mathrm{H} 2, \mathrm{~K} 1, \\
\mathrm{~K} 2, \mathrm{M}, \\
\mathrm{T} 1\end{array}$ \\
\hline & & Cheyenne & $\begin{array}{l}\text { thickening } \\
\text { agent }\end{array}$ & root & $\begin{array}{l}\text { dried and made } \\
\text { into a powder }\end{array}$ & $\mathrm{H} 1, \mathrm{M}$ \\
\hline
\end{tabular}




\begin{tabular}{|c|c|c|c|c|c|c|}
\hline \multicolumn{2}{|l|}{ Plant names } & \multirow{2}{*}{\begin{tabular}{|l} 
Cultural \\
source
\end{tabular}} & \multicolumn{3}{|c|}{ Plant information } & \multirow[t]{2}{*}{ Sources } \\
\hline Scientific & Common & & Uses & Parts used & Preparation & \\
\hline \multirow[t]{5}{*}{ P. esculentum cont. } & \multirow[t]{5}{*}{$\begin{array}{l}\text { Pomme de } \\
\text { prairie }\end{array}$} & \multirow[t]{2}{*}{ Cheyenne } & \multirow[t]{2}{*}{ food } & unknown & $\begin{array}{l}\text { dried plant } \\
\text { slices boiled and } \\
\text { sweetened to } \\
\text { make pudding } \\
\end{array}$ & $\mathrm{H} 1, \mathrm{M}$ \\
\hline & & & & root & eaten raw or dried & $\mathrm{H} 1, \mathrm{M}$ \\
\hline & & \multirow[t]{3}{*}{ MT Indian } & \multirow[t]{3}{*}{ food } & \multirow[t]{3}{*}{ root } & $\begin{array}{l}\text { dried and mashed } \\
\text { into bread, cake } \\
\text { thickener, or porridge }\end{array}$ & M \\
\hline & & & & & $\begin{array}{l}\text { eaten dried and } \\
\text { shredded or inner } \\
\text { root core eaten raw, } \\
\text { roasted, or boiled }\end{array}$ & M \\
\hline & & & & & $\begin{array}{l}\text { dried and stored or } \\
\text { crushed into powder }\end{array}$ & M \\
\hline $\begin{array}{l}\text { Psoralea hypogaea } \\
\text { Torr. \& A.Gray }\end{array}$ & $\begin{array}{l}\text { Little indian } \\
\text { breadroot }\end{array}$ & Cheyenne & food & root & $\begin{array}{l}\text { eaten raw or dried } \\
\text { and stored }\end{array}$ & M \\
\hline $\begin{array}{l}\text { Vicia americana } \\
\text { Willd. }\end{array}$ & $\begin{array}{l}\text { American } \\
\text { purple vetch }\end{array}$ & MT Indian & food & unknown & $\begin{array}{l}\text { cooked and eaten } \\
\text { for greens }\end{array}$ & $B, M$ \\
\hline \multicolumn{7}{|l|}{ Lamiaceae } \\
\hline $\begin{array}{l}\text { Agastache } \\
\text { foeniculum } \\
\text { (Pursh) Kuntze } \\
\end{array}$ & $\begin{array}{l}\text { Lavender } \\
\text { hyssop }\end{array}$ & Cheyenne & beverage & leaves & made into a tea & M \\
\hline $\begin{array}{l}\text { Lycopus asper } \\
\text { Greene }\end{array}$ & $\begin{array}{l}\text { Rough } \\
\text { bugleweed }\end{array}$ & Chippewa & food & unknown & dried or boiled & $M$ \\
\hline \multirow[t]{9}{*}{ Mentha arvensis L. } & \multirow[t]{9}{*}{ Wild mint } & Cheyenne & \multirow[t]{2}{*}{ beverage } & $\begin{array}{l}\text { leaves } \\
\text { and stem }\end{array}$ & $\begin{array}{l}\text { made into a tea } \\
\text { for flavoring }\end{array}$ & $\begin{array}{l}\mathrm{H} 2, \mathrm{~J} \\
\mathrm{~K} 2, \mathrm{M}\end{array}$ \\
\hline & & Cree & & unknown & made into a tea & $\begin{array}{l}\mathrm{H} 2, \mathrm{~J} \\
\mathrm{~K} 2, \mathrm{M}\end{array}$ \\
\hline & & Salish & insecticide & leaves & $\begin{array}{l}\text { powdered and } \\
\text { sprinkled on meat } \\
\text { and berries }\end{array}$ & $\begin{array}{l}\mathrm{H} 2, \mathrm{~J} \\
\mathrm{~K} 2, \mathrm{M}\end{array}$ \\
\hline & & \multirow[t]{2}{*}{ Kutenai } & tonic & unknown & infusion & $\begin{array}{l}\mathrm{H} 2, \mathrm{~J} \\
\mathrm{~K} 2, \mathrm{M}\end{array}$ \\
\hline & & & insecticide & leaves & $\begin{array}{l}\text { powdered and } \\
\text { sprinkled on meat } \\
\text { and berries }\end{array}$ & $\begin{array}{l}\mathrm{H} 2, \mathrm{~J} \\
\mathrm{~K} 2, \mathrm{M}\end{array}$ \\
\hline & & \multirow[t]{2}{*}{ Blackfeet } & spice & leaves & added to meat & $\begin{array}{l}\mathrm{H} 2, \mathrm{~J} \\
\mathrm{~K} 2, \mathrm{M} \\
\end{array}$ \\
\hline & & & beverage & unknown & $\begin{array}{l}\text { dried and used } \\
\text { to make tea }\end{array}$ & $\begin{array}{l}\mathrm{H} 2, \mathrm{~J} \\
\mathrm{~K} 2, \mathrm{M}\end{array}$ \\
\hline & & Ojibwa & beverage & unknown & made into a tea & $\begin{array}{l}\mathrm{H} 2, \mathrm{~J} \\
\mathrm{~K} 2, \mathrm{M}\end{array}$ \\
\hline & & MT Indian & beverage & unknown & unspecified & $\begin{array}{l}\mathrm{H} 2, \mathrm{~J} \\
\mathrm{~K} 2, \mathrm{M}\end{array}$ \\
\hline
\end{tabular}


Dexter et al. - Prehistoric Plant Use at Beaver Creek Rock Shelter,

Southwestern Montana, U.S.A.

\begin{tabular}{|c|c|c|c|c|c|c|}
\hline \multicolumn{2}{|l|}{ Plant names } & \multirow{2}{*}{$\begin{array}{l}\text { Cultural } \\
\text { source }\end{array}$} & \multicolumn{3}{|c|}{ Plant information } & \multirow[t]{2}{*}{ Sources } \\
\hline Scientific & Common & & Uses & Parts used & Preparation & \\
\hline \multirow[t]{4}{*}{ Monarda fistulosa L. } & \multirow[t]{4}{*}{ Beebalm } & \multirow[t]{2}{*}{ Blackfeet } & eating tool & flowerhead & $\begin{array}{l}\text { dried and used } \\
\text { like a spoon }\end{array}$ & $\mathrm{K} 1, \mathrm{M}$ \\
\hline & & & $\begin{array}{l}\text { food prep } \\
\text { tool }\end{array}$ & flowerhead & $\begin{array}{l}\text { dried and used to } \\
\text { apply water to a } \\
\text { green hide to make } \\
\text { it easier to scrape }\end{array}$ & $\mathrm{K} 1, \mathrm{M}$ \\
\hline & & \multirow[t]{2}{*}{ Salish } & preservative & leaves & $\begin{array}{l}\text { pulverized and } \\
\text { sprinkled on meat }\end{array}$ & $\mathrm{K} 1, \mathrm{M}$ \\
\hline & & & insecticide & leaves & $\begin{array}{l}\text { pulverized and } \\
\text { sprinkled on meat }\end{array}$ & $\mathrm{K} 1, \mathrm{M}$ \\
\hline \multicolumn{7}{|l|}{ Ranunculaceae } \\
\hline $\begin{array}{l}\text { Thalictrum } \\
\text { occidentale A.Gray }\end{array}$ & $\begin{array}{l}\text { Western } \\
\text { meadowrue }\end{array}$ & Blackfeet & spice & fruit & unspecified & $M$ \\
\hline
\end{tabular}

ceae and Apiaceae families. The primary method of food consumption was to eat the roots raw.

\section{Medicinal uses for pain and fever reduction}

Species in 5 of the 7 plant families are represented in the pain and fever-reducing category (Table 4). Of these 7 plant families, Apiaceae and Fabaceae represent the largest variety of plant species with 7 different species each. In treating pain and fevers, roots were the most utilized plant part. The most popular method for preparation was infusions with 19 entries.

Table 4. Plants implied from the archaeological record at Beaver Creek Rock Shelter, southwestern Montana, U.S.A. with ethnographic uses as medicines for pain and fever reduction. Information sources: (D) Densmore 1927, (G1) Gilmore 1977, (G2) Grinnell 1905, (G3) Grinnell 1972, (H1) Hart 1981, (H2) Hart 1992, (H3) Hoffman 1884, (H4) Hoffman 1891, (J1) Johnston 1970, (J2) Johnston 1987 (K1) Kindscher 1987, (K2) Kindscher 1992, (M) Moerman 1998, (S) Smith 1932, and (T) Taylor 1989.

\begin{tabular}{|c|c|c|c|c|c|c|}
\hline \multicolumn{2}{|l|}{ Plant names } & \multirow{2}{*}{$\begin{array}{l}\text { Cultural } \\
\text { source }\end{array}$} & \multicolumn{3}{|c|}{ Plant information } & \multirow[t]{2}{*}{ Sources } \\
\hline Scientific & Common & & Uses & Parts used & Preparation & \\
\hline \multicolumn{7}{|l|}{ Apiaceae } \\
\hline \multirow[t]{2}{*}{$\begin{array}{l}\text { Angelica dawsonii } \\
\text { S.Watson }\end{array}$} & \multirow[t]{2}{*}{$\begin{array}{l}\text { Dawson's } \\
\text { angelica }\end{array}$} & \multirow[t]{2}{*}{ Blackfeet } & antirheumatic & \multirow[t]{2}{*}{ root } & $\begin{array}{l}\text { poultice of } \\
\text { chewed roots }\end{array}$ & $M$ \\
\hline & & & analgesic & & $\begin{array}{l}\text { infusion for sore } \\
\text { armpits or groin }\end{array}$ & $M$ \\
\hline $\begin{array}{l}\text { Cicuta douglasii } \\
\text { (DC.) J.M.Coult. } \\
\text { \& Rose }\end{array}$ & $\begin{array}{l}\text { Western } \\
\text { water-hemlock }\end{array}$ & MT Indian & analgesic & root & unspecified & $M$ \\
\hline Cicuta maculata L. & $\begin{array}{l}\text { Spotted water- } \\
\text { hemlock }\end{array}$ & Cree & antirheumatic & root & $\begin{array}{l}\text { dried, powdered, \& } \\
\text { made into a liniment }\end{array}$ & $M$ \\
\hline $\begin{array}{l}\text { Ligusticum canbyi } \\
\text { J.M.Coult. \& Rose }\end{array}$ & $\begin{array}{l}\text { Canby's wild } \\
\text { lovage }\end{array}$ & Salish & $\begin{array}{l}\text { anticonvulsive } \\
\text { and headache }\end{array}$ & root & $\begin{array}{l}\text { chewed and } \\
\text { rubbed on body }\end{array}$ & $\mathrm{H} 2, \mathrm{M}$ \\
\hline $\begin{array}{l}\text { Lomatium } \\
\text { macrocarpum } \\
\text { (Hook. \& Arn.) } \\
\text { J.M.Coult. \& Rose }\end{array}$ & $\begin{array}{l}\text { Large-fruit } \\
\text { desert-parsley }\end{array}$ & Crow & antirheumatic & root & $\begin{array}{l}\text { poultice of root } \\
\text { shavings }\end{array}$ & $\mathrm{M}$ \\
\hline $\begin{array}{l}\text { Lomatium orientale } \\
\text { J.M.Coult. \& Rose }\end{array}$ & $\begin{array}{l}\text { Oriental } \\
\text { desert-parsley }\end{array}$ & Cheyenne & $\begin{array}{l}\text { anti- } \\
\text { inflammatory }\end{array}$ & $\begin{array}{l}\text { roots and } \\
\text { leaves }\end{array}$ & infusion & $\begin{array}{l}\text { G3, } \\
\mathrm{H} 2, \mathrm{M}\end{array}$ \\
\hline $\begin{array}{l}\text { Sanicula } \\
\text { marilandica L. }\end{array}$ & $\begin{array}{l}\text { Maryland black } \\
\text { snakeroot }\end{array}$ & Ojibwa & fever reducer & root & infusion & $M, S$ \\
\hline
\end{tabular}




\begin{tabular}{|c|c|c|c|c|c|c|}
\hline \multicolumn{2}{|l|}{ Plant names } & \multirow{2}{*}{$\begin{array}{l}\text { Cultural } \\
\text { source }\end{array}$} & \multicolumn{3}{|c|}{ Plant information } & \multirow[t]{2}{*}{ Sources } \\
\hline Scientific & Common & & Uses & Parts used & Preparation & \\
\hline \multicolumn{7}{|l|}{ Brassicaceae } \\
\hline \multirow{4}{*}{$\begin{array}{l}\text { Physaria } \\
\text { didymocarpa } \\
\text { (Hook.) A.Gray }\end{array}$} & \multirow[t]{4}{*}{$\begin{array}{l}\text { Common } \\
\text { twinpod }\end{array}$} & \multirow[t]{4}{*}{ Blackfeet } & analgesic & unknown & $\begin{array}{l}\text { plant chewed } \\
\text { for cramps }\end{array}$ & $M$ \\
\hline & & & antirheumatic & root & $\begin{array}{l}\text { infusion for } \\
\text { body aches }\end{array}$ & $M$ \\
\hline & & & antirheumatic & unknown & $\begin{array}{l}\text { strong infusion of } \\
\text { plant used as a } \\
\text { liniment on sprains }\end{array}$ & $M$ \\
\hline & & & $\begin{array}{l}\text { anti- } \\
\text { inflammatory }\end{array}$ & unknown & decoction of plant & $M$ \\
\hline \multicolumn{7}{|l|}{ Fabaceae } \\
\hline $\begin{array}{l}\text { Amorpha canescens } \\
\text { Pursh }\end{array}$ & Lead plant & Ojibwa & analgesic & root & decoction & $\mathrm{H} 3, \mathrm{M}$ \\
\hline $\begin{array}{l}\text { Astragalus } \\
\text { canadensis L. }\end{array}$ & $\begin{array}{l}\text { Canadian } \\
\text { milkvetch }\end{array}$ & Blackfeet & analgesic & root & $\begin{array}{l}\text { treats pediatric chest } \\
\text { aches by bathing } \\
\text { in the steam }\end{array}$ & $\mathrm{K} 2, \mathrm{M}$ \\
\hline $\begin{array}{l}\text { Astragalus } \\
\text { crassicarpus Nutt. }\end{array}$ & $\begin{array}{l}\text { Groundplum } \\
\text { milkvetch }\end{array}$ & Chippewa & $\begin{array}{l}\text { anticonvulsive } \\
\text { and headache }\end{array}$ & root & decoction & $D, M$ \\
\hline \multirow{2}{*}{$\begin{array}{l}\text { Glycyrrhiza lepidota } \\
\text { Pursh }\end{array}$} & \multirow[t]{2}{*}{ Wild licorice } & \multirow[t]{2}{*}{ Blackfeet } & analgesic & \multirow[t]{2}{*}{ root } & infusion & $M$ \\
\hline & & & antirheumatic & & infusion & $M$ \\
\hline $\begin{array}{l}\text { Pediomelum } \\
\text { argophyllum (Pursh) } \\
\text { J.W.Grimes }\end{array}$ & $\begin{array}{l}\text { Silvery } \\
\text { scurfpea }\end{array}$ & Cheyenne & fever reducer & $\begin{array}{l}\text { several } \\
\text { parts }\end{array}$ & $\begin{array}{l}\text { decoction or salve, } \\
\text { infusion of ground } \\
\text { leaves and stems }\end{array}$ & $\begin{array}{l}\text { G2, } \\
\text { G3, M }\end{array}$ \\
\hline $\begin{array}{l}\text { Pediomelum } \\
\text { esculentum } \\
\text { (Pursh) Rydb. }\end{array}$ & $\begin{array}{l}\text { Pomme de } \\
\text { prairie }\end{array}$ & Blackfeet & antirheumatic & root & $\begin{array}{l}\text { poultice of chewed } \\
\text { roots for sprains }\end{array}$ & $\begin{array}{l}\mathrm{H} 2, \mathrm{~K} 1 \\
\mathrm{~K} 2, \mathrm{M}, \mathrm{T}\end{array}$ \\
\hline \multirow{2}{*}{$\begin{array}{l}\text { Thermopsis } \\
\text { rhombifolia (Pursh) } \\
\text { Richardson }\end{array}$} & \multirow[t]{2}{*}{$\begin{array}{l}\text { Roundleaf } \\
\text { thermopsis }\end{array}$} & Cheyenne & \multirow[t]{2}{*}{$\begin{array}{l}\text { analgesic } \\
\text { antirheumatic }\end{array}$} & leaves & $\begin{array}{l}\text { dried, burned, } \\
\text { and inhaled }\end{array}$ & $\begin{array}{l}\text { G1, } \\
\mathrm{H} 1, \mathrm{M} \\
\end{array}$ \\
\hline & & MT Indian & & flower & dried and smoked & G1, M \\
\hline \multicolumn{7}{|l|}{ Lamiaceae } \\
\hline \multirow[t]{3}{*}{$\begin{array}{l}\text { Agastache } \\
\text { foeniculum } \\
\text { (Pursh) Kuntze }\end{array}$} & \multirow[t]{3}{*}{$\begin{array}{l}\text { Lavender } \\
\text { hyssop }\end{array}$} & \multirow[t]{2}{*}{ Cheyenne } & analgesic & \multirow[t]{2}{*}{ leaves } & $\begin{array}{l}\text { infusion used to } \\
\text { treat chest pain } \\
\text { due to coughing }\end{array}$ & $\begin{array}{l}\mathrm{G} 3, \\
\mathrm{~K} 2, \mathrm{M}\end{array}$ \\
\hline & & & fever reducer & & $\begin{array}{l}\text { powdered and } \\
\text { rubbed on the body }\end{array}$ & $\mathrm{H} 1, \mathrm{M}$ \\
\hline & & Chippewa & analgesic & root & infusion & $\mathrm{H} 1, \mathrm{M}$ \\
\hline \multirow[t]{3}{*}{ Mentha arvensis L. } & \multirow[t]{3}{*}{ Wild mint } & \multirow[t]{3}{*}{ Cree } & headache & \multirow[t]{2}{*}{ leaves } & infusion & $\begin{array}{l}\mathrm{H} 2, \\
\mathrm{~J} 1, \mathrm{M}\end{array}$ \\
\hline & & & fever reducer & & infusion & $\mathrm{D}, \mathrm{M}$ \\
\hline & & & toothache & $\begin{array}{l}\text { leafy stems } \\
\text { and flowers }\end{array}$ & poultice & $\mathrm{D}, \mathrm{M}$ \\
\hline
\end{tabular}


Dexter et al. - Prehistoric Plant Use at Beaver Creek Rock Shelter,

Southwestern Montana, U.S.A.

\begin{tabular}{|c|c|c|c|c|c|c|}
\hline \multicolumn{2}{|l|}{ Plant names } & \multirow{2}{*}{$\begin{array}{l}\text { Cultural } \\
\text { source }\end{array}$} & \multicolumn{3}{|c|}{ Plant information } & \multirow[t]{2}{*}{ Sources } \\
\hline Scientific & Common & & Uses & Parts used & Preparation & \\
\hline \multirow[t]{7}{*}{ M. arvensis cont. } & \multirow[t]{7}{*}{ Wild mint } & \multirow[t]{2}{*}{ Salish } & toothache & leaves & infusion & $\begin{array}{l}\mathrm{H} 2, \\
\mathrm{~J} 1, \mathrm{M}\end{array}$ \\
\hline & & & fever reducer & leaves & infusion & $\begin{array}{l}\mathrm{H} 2, \\
\mathrm{~J} 1, \mathrm{M}\end{array}$ \\
\hline & & \multirow[t]{2}{*}{ Kutenai } & antirheumatic & leaves & poultice & $\begin{array}{l}\mathrm{H} 2, \\
\mathrm{~J} 1, \mathrm{M}\end{array}$ \\
\hline & & & fever reducer & unknown & infusion & $\begin{array}{l}\mathrm{H} 2, \\
\mathrm{~J} 1, \mathrm{M}\end{array}$ \\
\hline & & Blackfeet & chest pain & leaves & unspecified & $\begin{array}{l}\mathrm{H} 2, \\
\mathrm{~J} 1, \mathrm{M} \\
\end{array}$ \\
\hline & & \multirow[t]{2}{*}{ Ojibwa } & stomach pain & unknown & infusion of plant & $\begin{array}{l}\mathrm{H} 2, \\
\mathrm{~J} 1, \mathrm{M}\end{array}$ \\
\hline & & & fever reducer & leaves & infusion & $\begin{array}{l}\mathrm{H} 2, \\
\mathrm{~J} 1, \mathrm{M}\end{array}$ \\
\hline \multirow[t]{4}{*}{ Monarda fistulosa L. } & \multirow[t]{4}{*}{ Beebalm } & Chippewa & headache & leaves & $\begin{array}{l}\text { chewed and } \\
\text { placed in nostrils }\end{array}$ & $M$ \\
\hline & & Salish & fever reducer & unknown & infusion & M \\
\hline & & \multirow[t]{2}{*}{ Ojibwa } & fever reducer & flower & infusion & $\mathrm{H} 4, \mathrm{M}$ \\
\hline & & & $\begin{array}{l}\text { anticonvulsive } \\
\text { and headache }\end{array}$ & unknown & infusion & $M$ \\
\hline \multicolumn{7}{|l|}{ Ranunculaceae } \\
\hline $\begin{array}{l}\text { Anemone } \\
\text { canadensis L. }\end{array}$ & $\begin{array}{l}\text { Canada } \\
\text { anemone }\end{array}$ & Ojibwa & analgesic & root & $\begin{array}{l}\text { decoction for } \\
\text { lumbar pain }\end{array}$ & $\mathrm{H} 4, \mathrm{M}$ \\
\hline $\begin{array}{l}\text { Anemone multifida } \\
\text { Poir. }\end{array}$ & $\begin{array}{l}\text { Pacific } \\
\text { anemone }\end{array}$ & Blackfeet & headache & seed head & $\begin{array}{l}\text { burn on hot coals } \\
\text { and inhale smoke }\end{array}$ & $\mathrm{J} 2, \mathrm{M}$ \\
\hline $\begin{array}{l}\text { Clematis } \\
\text { hirsutissima Pursh }\end{array}$ & Sugarbowls & MT Indian & headache & leaves & decoction & $\mathrm{H} 4, \mathrm{M}$ \\
\hline $\begin{array}{l}\text { Clematis } \\
\text { ligusticifolia Nutt. }\end{array}$ & $\begin{array}{l}\text { Western } \\
\text { virgin's-bower }\end{array}$ & Blackfeet & fever reducer & bark & unspecified & $\mathrm{J} 1, \mathrm{~J} 2, \mathrm{M}$ \\
\hline $\begin{array}{l}\text { Thalictrum } \\
\text { dasycarpum Fisch., } \\
\text { C.A.Mey. \& Avé-Lall. }\end{array}$ & $\begin{array}{l}\text { Purple } \\
\text { meadowrue }\end{array}$ & Ojibwa & fever reducer & root & infusion & $M, S$ \\
\hline
\end{tabular}

\section{Stomach-related medicinal use}

All 7 plant families were traditionally used for stomachrelated ailments with Apiaceae and Fabaceae dominating with 5 plant species each (Table 5). The most common uses were general stomach aids and antidiarrheals. Roots were the most common plant part used with infusions, the preferred method for plant preparation.

\section{$\underline{\text { Domestic uses }}$}

Species in 5 of the 7 plant families were utilized for traditional domestic purposes, including fragrance, hair care, cosmetics, decorations, dye, smoking, and weaving materials (Table 6). Among the 16 different plant species, the most common use was fragrance. Ethnographic literature has shown there is not one preferred type of plant part or method of preparation for domestic use.

\section{Medicinal uses for ear, nose, and throat ailments}

Species in 6 of the 7 plant families were used for treating ailments of the ears, eyes, throat, nose, and mouth (Table 7). The plant family with the most medicinal uses in this category is Apiaceae. Throat aids were the most commonly treated ailment, and infusions made primarily of roots were the preparation method of choice.

\section{$\underline{\text { Traditional medicinal skin care }}$}

The Fabaceae family has the largest number of plant species traditionally used for skin care, including general 
Table 5. Plants implied from the archaeological record at Beaver Creek Rock Shelter, southwestern Montana, U.S.A. with ethnographic uses as stomach-related medicines. Information sources: (G) Grinnell 1972, (H1) Hart 1981, (H2) Hart 1992, (H3) Hoffman 1891, (J) Johnston 1970, (K1) Kindscher 1987, (K2) Kinscher 1992, (M) Moerman 1998, (S) Scheinost 2010, and (T) Taylor 1989.

\begin{tabular}{|c|c|c|c|c|c|c|}
\hline \multicolumn{2}{|l|}{ Plant names } & \multirow{2}{*}{$\begin{array}{l}\text { Cultural } \\
\text { source }\end{array}$} & \multicolumn{3}{|c|}{\begin{tabular}{|l|} 
Plant information \\
\end{tabular}} & \multirow[t]{2}{*}{ Sources } \\
\hline Scientific & Common & & Uses & Parts used & Preparation & \\
\hline \multicolumn{7}{|l|}{ Apiaceae } \\
\hline \multirow{2}{*}{$\begin{array}{l}\text { Angelica dawsonii } \\
\text { S.Watson }\end{array}$} & \multirow{2}{*}{$\begin{array}{l}\text { Dawson's } \\
\text { angelica }\end{array}$} & \multirow[t]{2}{*}{ Blackfeet } & stomach aid & \multirow[t]{2}{*}{ root } & \multirow[t]{2}{*}{ infusion } & $M$ \\
\hline & & & $\begin{array}{l}\text { malnutrition } \\
\text { aid }\end{array}$ & & & $\mathrm{M}$ \\
\hline \multirow{3}{*}{$\begin{array}{l}\text { Cicuta douglasii } \\
\text { (DC.) J.M.Coult. } \\
\text { \& Rose }\end{array}$} & \multirow[t]{3}{*}{$\begin{array}{l}\text { Western } \\
\text { water-hemlock }\end{array}$} & Kutenai & emetic & root & $\begin{array}{l}\text { infusion mixed } \\
\text { with water }\end{array}$ & M \\
\hline & & \multirow[t]{2}{*}{ Salish } & cathartic & \multirow[t]{2}{*}{ unknown } & \multirow[t]{2}{*}{ unspecified } & M \\
\hline & & & emetic & & & M \\
\hline \multirow{2}{*}{$\begin{array}{l}\text { Lomatium orientale } \\
\text { J.M.Coult. \& Rose }\end{array}$} & \multirow{2}{*}{$\begin{array}{l}\text { Oriental } \\
\text { desert-parsley }\end{array}$} & \multirow[t]{2}{*}{ Cheyenne } & stomach aid & \multirow{2}{*}{$\begin{array}{l}\text { roots and } \\
\text { leaves }\end{array}$} & \multirow[t]{2}{*}{ infusion } & $\mathrm{H} 2, \mathrm{M}$ \\
\hline & & & antidiarrheal & & & $\mathrm{H} 2, \mathrm{M}$ \\
\hline $\begin{array}{l}\text { Osmorhiza } \\
\text { longistylis (Torr.) } \\
\text { DC. }\end{array}$ & $\begin{array}{l}\text { Smoother } \\
\text { sweet-cicely }\end{array}$ & Cheyenne & stomach aid & $\begin{array}{l}\text { leaves, } \\
\text { stem, } \\
\text { and root } \\
\end{array}$ & infusion & G, M \\
\hline \multirow{4}{*}{$\begin{array}{l}\text { Perideridia montana } \\
\text { (Blank.) Dorn }\end{array}$} & \multirow{4}{*}{$\begin{array}{l}\text { Gairdner's } \\
\text { yampah }\end{array}$} & \multirow[t]{4}{*}{ Blackfeet } & antidiarrheal & \multirow[t]{4}{*}{ root } & \multirow[t]{2}{*}{ infusion } & $M$ \\
\hline & & & antiemetic & & & M \\
\hline & & & diuretic & & \multirow[t]{2}{*}{ eaten } & $M$ \\
\hline & & & laxative & & & $\mathrm{M}$ \\
\hline \multicolumn{7}{|l|}{ Brassicaceae } \\
\hline \multirow[t]{3}{*}{$\begin{array}{l}\text { Physaria } \\
\text { didymocarpa } \\
\text { (Hook.) A.Gray }\end{array}$} & \multirow[t]{3}{*}{$\begin{array}{l}\text { Common } \\
\text { twinpod }\end{array}$} & \multirow[t]{3}{*}{ Blackfeet } & stomach aid & unknown & $\begin{array}{l}\text { decoction that } \\
\text { expands the stomach } \\
\text { so food can be } \\
\text { eaten without pain } \\
\text { by someone who } \\
\text { has not eaten } \\
\text { for an extended } \\
\text { period of time }\end{array}$ & M \\
\hline & & & $\begin{array}{l}\text { stomach } \\
\text { cramps }\end{array}$ & unknown & chewed & M \\
\hline & & & stomach pain & leaves & infusion & $M$ \\
\hline \multicolumn{7}{|l|}{ Cyperaceae } \\
\hline $\begin{array}{l}\text { Schoenoplectus } \\
\text { acutus (Muhl. ex } \\
\text { Bigelow) Á.Löve } \\
\text { \& D.Löve }\end{array}$ & $\begin{array}{l}\text { Hardstem } \\
\text { bulrush }\end{array}$ & MT Indian & dietary aid & root & $\begin{array}{l}\text { chewed to prevent } \\
\text { dehydration }\end{array}$ & $\mathrm{J}, \mathrm{M}$ \\
\hline \multicolumn{7}{|l|}{ Fabaceae } \\
\hline $\begin{array}{l}\text { Amorpha canescens } \\
\text { Pursh }\end{array}$ & Lead plant & Ojibwa & stomach aid & root & decoction & $\mathrm{H} 3, \mathrm{M}$ \\
\hline \multirow{2}{*}{$\begin{array}{l}\text { Astragalus } \\
\text { americanus (Hook.) } \\
\text { M.E.Jones }\end{array}$} & American & Cree & stomach pain & root & chewed & $M$ \\
\hline & milkvetch & & stomach flu & & & M \\
\hline Dalea candida Willd. & $\begin{array}{l}\text { White prairie } \\
\text { clover }\end{array}$ & Blackfeet & antidiarrheal & root & $\begin{array}{l}\text { dried and made } \\
\text { into a tea }\end{array}$ & K1 \\
\hline
\end{tabular}


Dexter et al. - Prehistoric Plant Use at Beaver Creek Rock Shelter,

Southwestern Montana, U.S.A.

\begin{tabular}{|c|c|c|c|c|c|c|}
\hline \multicolumn{2}{|l|}{ Plant names } & \multirow{2}{*}{$\begin{array}{l}\text { Cultural } \\
\text { source }\end{array}$} & \multicolumn{3}{|c|}{ Plant information } & \multirow[t]{2}{*}{ Sources } \\
\hline Scientific & Common & & Uses & Parts used & Preparation & \\
\hline \multirow[t]{2}{*}{$\begin{array}{l}\text { Glycyrrhiza lepidota } \\
\text { Pursh }\end{array}$} & \multirow[t]{2}{*}{ Wild licorice } & \multirow[t]{2}{*}{ Cheyenne } & antidiarrheal & \multirow[t]{2}{*}{$\begin{array}{l}\text { roots and } \\
\text { leaves }\end{array}$} & \multirow[t]{2}{*}{ infusion } & $\begin{array}{l}\mathrm{H} 1 \\
\mathrm{H} 2, \mathrm{M}\end{array}$ \\
\hline & & & stomach aid & & & $\begin{array}{l}\mathrm{H} 1, \\
\mathrm{H} 2, \mathrm{M}\end{array}$ \\
\hline \multirow{4}{*}{$\begin{array}{l}\text { Pediomelum } \\
\text { esculentum (Pursh) } \\
\text { Rydb. }\end{array}$} & \multirow[t]{4}{*}{$\begin{array}{l}\text { Pomme de } \\
\text { prairie }\end{array}$} & \multirow[t]{3}{*}{ Blackfeet } & gastroenteritis & \multirow[t]{3}{*}{ root } & dried & $\begin{array}{l}\mathrm{K} 1, \mathrm{~K} 2 \\
\mathrm{M}, \mathrm{T}\end{array}$ \\
\hline & & & $\begin{array}{l}\text { bowel } \\
\text { complaints }\end{array}$ & & chewed & $\begin{array}{l}\mathrm{K} 1, \mathrm{~K} 2 \\
\mathrm{M}, \mathrm{T}\end{array}$ \\
\hline & & & colic & & $\begin{array}{l}\text { chewed and blown } \\
\text { into baby's rectum }\end{array}$ & $\begin{array}{l}\mathrm{K} 1, \mathrm{~K} 2 \\
\mathrm{M}, \mathrm{T}\end{array}$ \\
\hline & & Cheyenne & antidiarrheal & unknown & unspecified & $\mathrm{H} 1, \mathrm{M}$ \\
\hline \multicolumn{7}{|l|}{ Geraniaceae } \\
\hline $\begin{array}{l}\text { Geranium } \\
\text { viscosissimum } \\
\text { Fisch. \& C.A.Mey. }\end{array}$ & $\begin{array}{l}\text { Sticky } \\
\text { geranium }\end{array}$ & Blackfeet & $\begin{array}{l}\text { malnutrition } \\
\text { aid }\end{array}$ & leaves & $\begin{array}{l}\text { infusion eaten and } \\
\text { applied to the heads } \\
\text { of two women with } \\
\text { large heads due to } \\
\text { malnutrition. Both } \\
\text { women experienced } \\
\text { relief and soon } \\
\text { after died. }\end{array}$ & $M, S$ \\
\hline \multicolumn{7}{|l|}{ Lamiaceae } \\
\hline \multirow[t]{2}{*}{ Mentha arvensis L. } & \multirow[t]{2}{*}{ Wild mint } & Cheyenne & antiemetic & $\begin{array}{l}\text { leaves } \\
\text { and stem }\end{array}$ & $\begin{array}{l}\text { ground infusion } \\
\text { or boiled }\end{array}$ & $\mathrm{K} 2, \mathrm{M}$ \\
\hline & & MT Indian & relieves gas & unknown & $\begin{array}{l}\text { steeped in water } \\
\text { and drunk }\end{array}$ & $\mathrm{K} 2, \mathrm{M}$ \\
\hline \multirow[t]{2}{*}{ Monarda fistulosa L. } & \multirow[t]{2}{*}{ Beebalm } & Blackfeet & emetic & unknown & infusion & $\mathrm{K} 2, \mathrm{M}$ \\
\hline & & Ojibwa & stomach aid & root & decoction & $\mathrm{K} 2, \mathrm{M}$ \\
\hline Prunella vulgaris L. & Self-heal & Chippewa & cathartic & root & compound decoction & $M$ \\
\hline Stachys palustris L. & $\begin{array}{l}\text { Marsh } \\
\text { hedge-nettle }\end{array}$ & Chippewa & $\begin{array}{l}\text { gastro- } \\
\text { intestinal aid }\end{array}$ & leaves & $\begin{array}{l}\text { fresh or dried } \\
\text { infusion for } \\
\text { sudden colic }\end{array}$ & $M$ \\
\hline \multicolumn{7}{|l|}{ Ranunculaceae } \\
\hline \multirow[t]{2}{*}{$\begin{array}{l}\text { Actaea rubra (Aiton) } \\
\text { Willd. }\end{array}$} & \multirow[t]{2}{*}{$\begin{array}{l}\text { Red } \\
\text { baneberry }\end{array}$} & Cheyenne & dietary aid & root & $\begin{array}{l}\text { decoction for } \\
\text { improving appetite }\end{array}$ & $\begin{array}{l}\mathrm{H} 1 \\
\mathrm{H} 2, \mathrm{M}\end{array}$ \\
\hline & & Ojibwa & stomach aid & root & eaten & $\begin{array}{l}\mathrm{H} 1, \\
\mathrm{H} 2, \mathrm{M}\end{array}$ \\
\hline $\begin{array}{l}\text { Delphinium bicolor } \\
\text { Nutt. }\end{array}$ & Little larkspur & Blackfeet & antidiarrheal & unknown & infusion & $M$ \\
\hline
\end{tabular}


Table 6. Plants implied from the archaeological record at Beaver Creek Rock Shelter, southwestern Montana, U.S.A. with ethnographic uses for domestic purposes. Information sources: (B) Blankinship 1905, (G1) Gilmore 1977, (G2) Grinnell 1972, (H1) Hart 1981, (H2) Hart 1992, (J) Johnston 1970, (K1) Kindscher 1987, (K2) Kindscher 1992, (M) Moerman 1998, (S) Smith 1932, (T1) Taylor 1989, and (T2) Turner 1971.

\begin{tabular}{|c|c|c|c|c|c|c|}
\hline \multicolumn{2}{|l|}{ Plant names } & \multirow{2}{*}{$\begin{array}{l}\text { Cultural } \\
\text { source }\end{array}$} & \multicolumn{3}{|c|}{ Plant information } & \multirow[t]{2}{*}{ Sources } \\
\hline Scientific & Common & & Uses & Parts used & Preparation & \\
\hline \multicolumn{7}{|l|}{ Apiaceae } \\
\hline Cicuta maculata L. & $\begin{array}{l}\text { Spotted water- } \\
\text { hemlock }\end{array}$ & Chippewa & smoke & seed & mixed with tobacco & $M$ \\
\hline \multirow{3}{*}{$\begin{array}{l}\text { Ligusticum canbyi } \\
\text { J.M.Coult. \& Rose }\end{array}$} & \multirow{3}{*}{$\begin{array}{l}\text { Canby's wild } \\
\text { lovage }\end{array}$} & \multirow[t]{2}{*}{ Crow } & fragrance & root & shavings on live coals & $\mathrm{H} 2, \mathrm{M}$ \\
\hline & & & smoke & root & $\begin{array}{l}\text { shavings added } \\
\text { to tobacco }\end{array}$ & $\mathrm{H} 2, \mathrm{M}$ \\
\hline & & Salish & hair soap & root & $\begin{array}{l}\text { mixed with } \\
\text { buckbrush leaves }\end{array}$ & $\mathrm{H} 2, \mathrm{M}$ \\
\hline \multirow{2}{*}{$\begin{array}{l}\text { Osmorhiza } \\
\text { occidentalis } \\
\text { (Nutt.) Torr. }\end{array}$} & \multirow[t]{2}{*}{$\begin{array}{l}\text { Western } \\
\text { sweet-cicely }\end{array}$} & \multirow[t]{2}{*}{ Blackfeet } & dye & stem & $\begin{array}{l}\text { mixed with ochre and } \\
\text { applied to robes }\end{array}$ & M \\
\hline & & & fragrance & root & $\begin{array}{l}\text { used as perfume for } \\
\text { clothes and diapers }\end{array}$ & $M$ \\
\hline \multicolumn{7}{|l|}{ Cyperaceae } \\
\hline \multirow{2}{*}{$\begin{array}{l}\text { Amphiscirpus } \\
\text { nevadensis } \\
\text { (S.Watson) } \\
\text { Oteng-Yeb. } \\
\end{array}$} & \multirow[t]{2}{*}{$\begin{array}{l}\text { Nevada } \\
\text { bulrush }\end{array}$} & \multirow[t]{2}{*}{ Cheyenne } & $\begin{array}{l}\text { weaving } \\
\text { material }\end{array}$ & stem & $\begin{array}{l}\text { woven and used for } \\
\text { rugs, and bedding }\end{array}$ & $\mathrm{G} 2, \mathrm{M}$ \\
\hline & & & ceremonial & unknown & Sundance ceremony & G2, M \\
\hline \multirow[t]{2}{*}{$\begin{array}{l}\text { Carex nebraskensis } \\
\text { Dewey }\end{array}$} & \multirow[t]{2}{*}{$\begin{array}{l}\text { Nebraska } \\
\text { sedge }\end{array}$} & Cheyenne & ceremonial & unknown & $\begin{array}{l}\text { Sundance and } \\
\text { Massaum ceremonies }\end{array}$ & $\mathrm{H} 1, \mathrm{~J}, \mathrm{M}$ \\
\hline & & Blackfeet & ceremonial & $\begin{array}{l}\text { leaves and } \\
\text { grass }\end{array}$ & $\begin{array}{l}\text { tied around buffalo } \\
\text { skulls because it } \\
\text { is thought to be } \\
\text { a favorite food } \\
\text { of the buffalo } \\
\end{array}$ & $\mathrm{H} 1, \mathrm{~J}, \mathrm{M}$ \\
\hline $\begin{array}{l}\text { Schoenoplectus } \\
\text { acutus (Muhl. ex } \\
\text { Bigelow) Á.Löve } \\
\text { \& D.Löve } \\
\end{array}$ & $\begin{array}{l}\text { Hardstem } \\
\text { bulrush }\end{array}$ & MT Indian & $\begin{array}{l}\text { weaving } \\
\text { material }\end{array}$ & stem & $\begin{array}{l}\text { used to make mats, } \\
\text { rugs and bedding }\end{array}$ & $\mathrm{B}, \mathrm{J} 1, \mathrm{M}$ \\
\hline $\begin{array}{l}\text { Schoenoplectus } \\
\text { americanus } \\
\text { (Pers.) Volkart }\end{array}$ & $\begin{array}{l}\text { Three-square } \\
\text { bulrush }\end{array}$ & Salish & $\begin{array}{l}\text { weaving } \\
\text { material }\end{array}$ & leaves & $\begin{array}{l}\text { sun-dried and used } \\
\text { to make mats, rugs, } \\
\text { and bedding }\end{array}$ & $\mathrm{M}, \mathrm{T} 2$ \\
\hline $\begin{array}{l}\text { Scirpus cyperinus } \\
\text { (L.) Kunth }\end{array}$ & Woolgrass & Ojibwa & $\begin{array}{l}\text { weaving } \\
\text { material }\end{array}$ & rushes & $\begin{array}{l}\text { used to make storage } \\
\text { bags and mats }\end{array}$ & $M$ \\
\hline \multicolumn{7}{|l|}{ Fabaceae } \\
\hline $\begin{array}{l}\text { Oxytropis sericea } \\
\text { Torr. \& A.Gray }\end{array}$ & \begin{tabular}{|l} 
White \\
locoweed
\end{tabular} & Blackfeet & decoration & stem & \begin{tabular}{|l|} 
jewelry on \\
headdresses for kids
\end{tabular} & $M$ \\
\hline $\begin{array}{l}\text { Psoralea esculenta } \\
\text { Pursh }\end{array}$ & $\begin{array}{l}\text { Pomme- } \\
\text { de-prairie }\end{array}$ & Blackfeet & decoration & root & $\begin{array}{l}\text { dried pieces used } \\
\text { on clothing }\end{array}$ & $\begin{array}{l}\mathrm{H} 2, \mathrm{~K} 1 \\
\mathrm{~K} 2, \mathrm{M} \\
\mathrm{T} 1\end{array}$ \\
\hline $\begin{array}{l}\text { Psoralea lanceolata } \\
\text { Pursh }\end{array}$ & $\begin{array}{l}\text { Lance-leaf } \\
\text { scurfpea }\end{array}$ & Cheyenne & ceremonial & unknown & $\begin{array}{l}\text { used for unspecified } \\
\text { ceremonies }\end{array}$ & $\mathrm{G} 2, \mathrm{M}$ \\
\hline $\begin{array}{l}\text { Thermopsis } \\
\text { rhombifolia (Pursh) } \\
\text { Richardson }\end{array}$ & $\begin{array}{l}\text { Roundleaf } \\
\text { thermopsis }\end{array}$ & Blackfeet & dye & $\begin{array}{l}\text { flower } \\
\text { petals }\end{array}$ & $\begin{array}{l}\text { rubbed on arrow shaft } \\
\text { for yellow coloring }\end{array}$ & $M$ \\
\hline
\end{tabular}


Dexter et al. - Prehistoric Plant Use at Beaver Creek Rock Shelter,

Southwestern Montana, U.S.A.

\begin{tabular}{|c|c|c|c|c|c|c|}
\hline \multicolumn{2}{|l|}{ Plant names } & \multirow{2}{*}{$\begin{array}{l}\text { Cultural } \\
\text { source }\end{array}$} & \multicolumn{3}{|c|}{ Plant information } & \multirow[t]{2}{*}{ Sources } \\
\hline Scientific & Common & & Uses & Parts used & Preparation & \\
\hline \multicolumn{7}{|l|}{ Lamiaceae } \\
\hline \multirow[t]{3}{*}{ Mentha arvensis L. } & \multirow[t]{3}{*}{ Wild mint } & \multirow[t]{2}{*}{ Cheyenne } & hair oil & unknown & decoction of plant & $\mathrm{K} 2, \mathrm{M}$ \\
\hline & & & fragrance & $\begin{array}{l}\text { leaves } \\
\text { and stem }\end{array}$ & used as perfume & $\mathrm{K} 2, \mathrm{M}$ \\
\hline & & MT Indian & fragrance & unknown & for the home & $\mathrm{K} 2, \mathrm{M}$ \\
\hline \multirow[t]{3}{*}{ Monarda fistulosa L. } & \multirow[t]{3}{*}{ Beebalm } & Crow & fragrance & unknown & $\begin{array}{l}\text { plant mixed with other } \\
\text { plants and beaver } \\
\text { castor oil for use on } \\
\text { hair, body, \& clothing }\end{array}$ & $M$ \\
\hline & & Kutenai & fragrance & leaves & $\begin{array}{l}\text { placed on hot rocks for } \\
\text { scent in sweat house }\end{array}$ & $M$ \\
\hline & & MT Indian & fragrance & unknown & $\begin{array}{l}\text { used as perfume for } \\
\text { hair, body, and home }\end{array}$ & $M$ \\
\hline \multicolumn{7}{|l|}{ Ranunculaceae } \\
\hline \multirow[t]{2}{*}{$\begin{array}{l}\text { Delphinium bicolor } \\
\text { Nutt. }\end{array}$} & \multirow[t]{2}{*}{ Little larkspur } & \multirow[t]{2}{*}{ Blackfeet } & $\begin{array}{l}\text { shine and } \\
\text { straighten } \\
\text { hair }\end{array}$ & unknown & infusion & $M$ \\
\hline & & & dye & flower & $\begin{array}{l}\text { used to dye quills } \\
\text { light blue }\end{array}$ & $M$ \\
\hline $\begin{array}{l}\text { Ranunculus } \\
\text { pensylvanicus L.f. }\end{array}$ & $\begin{array}{l}\text { Bristly } \\
\text { crowfoot }\end{array}$ & Ojibwa & dye & entire plant & boiled for red coloring & $M, S$ \\
\hline \multirow{2}{*}{$\begin{array}{l}\text { Thalictrum } \\
\text { dasycarpum Fisch., } \\
\text { C.A.Mey. \& Avé-Lall. }\end{array}$} & \multirow{2}{*}{$\begin{array}{l}\text { Purple } \\
\text { meadowrue }\end{array}$} & Blackfeet & fragrance & fruit & unspecified & G1, J, M \\
\hline & & MT Indian & fragrance & fruit & stored for smell & $\mathrm{G} 1, \mathrm{~J}, \mathrm{M}$ \\
\hline \multirow[t]{4}{*}{$\begin{array}{l}\text { Thalictrum } \\
\text { occidentale A.Gray }\end{array}$} & \multirow[t]{4}{*}{$\begin{array}{l}\text { Western } \\
\text { meadowrue }\end{array}$} & \multirow[t]{4}{*}{ Blackfeet } & cosmetic & fruit & $\begin{array}{l}\text { powdered and } \\
\text { mixed with water } \\
\end{array}$ & $\mathrm{J}, \mathrm{M}$ \\
\hline & & & insecticide & $\begin{array}{l}\text { fruit, seeds, } \\
\text { leaves }\end{array}$ & unspecified & $\mathrm{J}, \mathrm{M}$ \\
\hline & & & fragrance & fruit & $\begin{array}{l}\text { dried and placed } \\
\text { in buckskin bags }\end{array}$ & $\mathrm{J}, \mathrm{M}$ \\
\hline & & & paint & fruit & $\begin{array}{l}\text { crushed and used } \\
\text { as paint for robes }\end{array}$ & \\
\hline
\end{tabular}

Table 7. Plants implied from the archaeological record at Beaver Creek Rock Shelter, southwestern Montana, U.S.A. with ethnographic uses for ear, nose and throat ailments. Information sources: (B) Blankinship 1905, (H) Hart 1992, (J) Johnston 1970, (K1) Kindscher 1987, (K2) Kindscher 1992, (M) Moerman 1998, (S) Smith 1932, and (T) Taylor 1989.

\begin{tabular}{|c|c|c|c|c|c|c|}
\hline \multicolumn{2}{|l|}{ Plant names } & \multirow{2}{*}{$\begin{array}{l}\text { Cultural } \\
\text { source }\end{array}$} & \multicolumn{3}{|c|}{ Plant information } & \multirow[t]{2}{*}{ Sources } \\
\hline Scientific & Common & & Uses & Parts used & Preparation & \\
\hline \multicolumn{7}{|l|}{ Apiaceae } \\
\hline $\begin{array}{l}\text { Ligusticum canbyi } \\
\text { J.M. Coult. \& Rose }\end{array}$ & $\begin{array}{l}\text { Canby's wild } \\
\text { lovage }\end{array}$ & Crow & ear aid & root & infusion & $\mathrm{H} 2, \mathrm{M}$ \\
\hline $\begin{array}{l}\text { Osmorhiza chilensis } \\
\text { Hook. \& Arn. }\end{array}$ & $\begin{array}{l}\text { Chilean } \\
\text { sweet-cicely }\end{array}$ & Blackfeet & throat aid & root & hot drink & $M$ \\
\hline $\begin{array}{l}\text { Osmorhiza longistylis } \\
\text { (Torr.) DC. }\end{array}$ & $\begin{array}{l}\text { Smoother } \\
\text { sweet-cicely }\end{array}$ & Ojibwa & throat aid & root & infusion & $M, S$ \\
\hline
\end{tabular}




\begin{tabular}{|c|c|c|c|c|c|c|}
\hline \multicolumn{2}{|l|}{ Plant names } & \multirow{2}{*}{$\begin{array}{l}\text { Cultural } \\
\text { source }\end{array}$} & \multicolumn{3}{|c|}{ Plant information } & \multirow[t]{2}{*}{ Sources } \\
\hline Scientific & Common & & Uses & Parts used & Preparation & \\
\hline \multirow{2}{*}{$\begin{array}{l}\text { Osmorhiza } \\
\text { occidentalis } \\
\text { (Nutt.) Torr. }\end{array}$} & \multirow{2}{*}{$\begin{array}{l}\text { Western } \\
\text { sweet-cicely }\end{array}$} & \multirow[t]{2}{*}{ Blackfeet } & eye aid & root & infusion & $\mathrm{M}$ \\
\hline & & & nose aid & root & infustion & M \\
\hline $\begin{array}{l}\text { Perideridia montana } \\
\text { (Blank.) Dorn }\end{array}$ & $\begin{array}{l}\text { Gairdner's } \\
\text { yampah }\end{array}$ & Blackfeet & throat aid & root & infusion & $M$ \\
\hline $\begin{array}{l}\text { Peucedanum } \\
\text { macrocarpum Nutt. }\end{array}$ & $\begin{array}{l}\text { Large-fruit } \\
\text { desert-parsley }\end{array}$ & Crow & throat aid & root & chewed for the juice & M \\
\hline \multicolumn{7}{|l|}{ Brassicaceae } \\
\hline \multirow{4}{*}{$\begin{array}{l}\text { Physaria } \\
\text { didymocarpa } \\
\text { (Hook.) A.Gray }\end{array}$} & \multirow[t]{4}{*}{$\begin{array}{l}\text { Common } \\
\text { twinpod }\end{array}$} & \multirow[t]{4}{*}{ Blackfeet } & ear aid & leaves & $\begin{array}{l}\text { infusion for ear } \\
\text { infections }\end{array}$ & M \\
\hline & & & eye aid & leaves & $\begin{array}{l}\text { infusion for } \\
\text { bloodshot eyes } \\
\end{array}$ & M \\
\hline & & & throat aid & unknown & chewed & $\mathrm{M}$ \\
\hline & & & oral aid & leaves & $\begin{array}{l}\text { clenched between } \\
\text { the teeth to treat } \\
\text { toothaches }\end{array}$ & M \\
\hline \multicolumn{7}{|l|}{ Fabaceae } \\
\hline \multirow{2}{*}{$\begin{array}{l}\text { Glycyrrhiza lepidota } \\
\text { Pursh }\end{array}$} & \multirow[t]{2}{*}{ Wild licorice } & Blackfeet & throat aid & root & infusion & $\mathrm{H}, \mathrm{M}$ \\
\hline & & MT Indian & throat aid & root & $\begin{array}{l}\text { chewed for the juice } \\
\text { to strengthen the } \\
\text { throat for singing }\end{array}$ & $\mathrm{H}, \mathrm{M}$ \\
\hline $\begin{array}{l}\text { Oxytropis lagopus } \\
\text { Nutt. }\end{array}$ & $\begin{array}{l}\text { Hare's-foot } \\
\text { locoweed }\end{array}$ & Blackfeet & throat aid & leaves & chewed & $\mathrm{K} 2, \mathrm{M}$ \\
\hline $\begin{array}{l}\text { Oxytropis sericea } \\
\text { Torr. \& A.Gray }\end{array}$ & $\begin{array}{l}\text { White } \\
\text { locoweed }\end{array}$ & Blackfeet & ear aid & leaves & infusion & $\mathrm{K} 2, \mathrm{M}$ \\
\hline \multirow[t]{4}{*}{$\begin{array}{l}\text { Psoralea esculenta } \\
\text { Pursh }\end{array}$} & \multirow[t]{4}{*}{$\begin{array}{l}\text { Pomme- } \\
\text { de-prairie }\end{array}$} & \multirow[t]{4}{*}{ Blackfeet } & ear aid & root & $\begin{array}{l}\text { chewed root spittle } \\
\text { to remove matter }\end{array}$ & $\begin{array}{l}\mathrm{K} 1, \mathrm{~K} 2 \\
\mathrm{H}, \mathrm{M}, \mathrm{T}\end{array}$ \\
\hline & & & eye aid & root & $\begin{array}{l}\text { chewed root spittle } \\
\text { to remove matter }\end{array}$ & $\begin{array}{l}\mathrm{K} 1, \mathrm{~K} 2 \\
\mathrm{H}, \mathrm{M}, \mathrm{T}\end{array}$ \\
\hline & & & throat aid & root & chewed & $\begin{array}{l}\mathrm{K} 1, \mathrm{~K} 2 \\
\mathrm{H}, \mathrm{M}, \mathrm{T}\end{array}$ \\
\hline & & & oral aid & root & chewed for teething & $\begin{array}{l}\mathrm{K} 1, \mathrm{~K} 2 \\
\mathrm{H}, \mathrm{M}, \mathrm{T}\end{array}$ \\
\hline \multicolumn{7}{|l|}{ Geraniaceae } \\
\hline $\begin{array}{l}\text { Geranium } \\
\text { viscosissimum } \\
\text { Fisch. \& C.A.Mey. }\end{array}$ & $\begin{array}{l}\text { Sticky } \\
\text { geranium }\end{array}$ & Blackfeet & eye aid & leaves & infusion & M \\
\hline \multicolumn{7}{|l|}{ Lamiaceae } \\
\hline Mentha arvensis L. & Wild mint & Cree & oral aid & flower & $\begin{array}{l}\text { ground, mixed with } \\
\text { yarrow, put into a } \\
\text { cloth, moistened, } \\
\text { and rubbed on the } \\
\text { gum to remove pus } \\
\end{array}$ & $\mathrm{D}, \mathrm{M}$ \\
\hline Monarda fistulosa L. & Beebalm & Blackfeet & throat aid & root & $\begin{array}{l}\text { chewed for } \\
\text { swollen glands }\end{array}$ & $\mathrm{J}, \mathrm{K} 2, \mathrm{M}$ \\
\hline
\end{tabular}


Dexter et al. - Prehistoric Plant Use at Beaver Creek Rock Shelter,

Southwestern Montana, U.S.A.

\begin{tabular}{|c|c|c|c|c|c|c|}
\hline \multicolumn{2}{|l|}{ Plant names } & \multirow{2}{*}{$\begin{array}{l}\text { Cultural } \\
\text { source }\end{array}$} & \multicolumn{3}{|c|}{ Plant information } & \multirow[t]{2}{*}{ Sources } \\
\hline Scientific & Common & & Uses & Parts used & Preparation & \\
\hline \multirow[t]{3}{*}{ M. fistulosa cont. } & \multirow[t]{3}{*}{ Beebalm } & Blackfeet & eye aid & blossoms & solution for eye wash & $\mathrm{J}, \mathrm{K} 2, \mathrm{M}$ \\
\hline & & \multirow[t]{2}{*}{ Salish } & oral aid & unknown & $\begin{array}{l}\text { unspecified for } \\
\text { toothaches }\end{array}$ & $\mathrm{J}, \mathrm{K} 2, \mathrm{M}$ \\
\hline & & & eye aid & unknown & solution for soreness & $\mathrm{J}, \mathrm{K} 2, \mathrm{M}$ \\
\hline Prunella vulgaris $\mathrm{L}$. & Self-heal & Blackfeet & eye aid & unknown & $\begin{array}{l}\text { infusion made into } \\
\text { a wash to keep the } \\
\text { eye moist and cold }\end{array}$ & $M$ \\
\hline \multicolumn{7}{|l|}{ Ranunculaceae } \\
\hline $\begin{array}{l}\text { Anemone canadensis } \\
\text { L. }\end{array}$ & $\begin{array}{l}\text { Canada } \\
\text { anemone }\end{array}$ & Ojibwa & throat aid & root & eaten to sing well & $M, S$ \\
\hline \multirow{2}{*}{$\begin{array}{l}\text { Clematis ligusticifolia } \\
\text { Nutt. }\end{array}$} & \multirow{2}{*}{$\begin{array}{l}\text { Western } \\
\text { virgin's-bower }\end{array}$} & MT Indian & throat aid & unknown & chewed & $\mathrm{B}, \mathrm{M}$ \\
\hline & & Blackfeet & throat aid & foliage & unspecified & $M$ \\
\hline
\end{tabular}

skin aids, burn dressings, and diaper rash (Table 8). Ethnographic literature indicates general skin aids were the most commonly used remedy. Infusions, made primarily with roots, were the most widespread practice for preparing the plants.

\section{$\underline{\text { Traditional cough and cold remedies }}$}

Species in 6 of the 7 plant families were traditionally used as cough and cold remedies (Table 9). The majority of the species were used to treat colds. Infusions, made primar-

Table 8. Plants implied from the archaeological record at Beaver Creek Rock Shelter, southwestern Montana, U.S.A. with ethnographic uses for skin care. Information sources: (B) Blankinship 1905, (H1) Hart 1981, (H2) Hart 1992, (K1) Kindscher 1987, (K2) Kindscher 1992, (M) Moerman 1998, and (T) Taylor 1989.

\begin{tabular}{|c|c|c|c|c|c|c|}
\hline \multicolumn{2}{|l|}{ Plant names } & \multirow{2}{*}{$\begin{array}{l}\text { Cultural } \\
\text { source }\end{array}$} & \multicolumn{3}{|c|}{ Plant information } & \multirow[t]{2}{*}{ Sources } \\
\hline Scientific & Common & & Uses & Parts used & Preparation & \\
\hline \multicolumn{7}{|l|}{ Apiaceae } \\
\hline $\begin{array}{l}\text { Angelica dawsonii } \\
\text { S.Watson }\end{array}$ & $\begin{array}{l}\text { Dawson's } \\
\text { angelica }\end{array}$ & Blackfeet & skin aid & root & $\begin{array}{l}\text { poultice of } \\
\text { chewed roots }\end{array}$ & $M$ \\
\hline $\begin{array}{l}\text { Cicuta douglasii } \\
\text { (DC.) J.M.Coult. } \\
\text { \& Rose }\end{array}$ & $\begin{array}{l}\text { Western } \\
\text { water-hemlock }\end{array}$ & Kutenai & skin aid & root & pounded & $M$ \\
\hline $\begin{array}{l}\text { Osmorhiza } \\
\text { occidentalis } \\
\text { (Nutt.) Torr. }\end{array}$ & $\begin{array}{l}\text { Western } \\
\text { sweet-cicely }\end{array}$ & Blackfeet & skin aid & root & infusion & $M$ \\
\hline $\begin{array}{l}\text { Perideridia montana } \\
\text { (Blank.) Dorn }\end{array}$ & $\begin{array}{l}\text { Gairdner's } \\
\text { yampah }\end{array}$ & Blackfeet & skin aid & root & infusion & $M$ \\
\hline \multicolumn{7}{|l|}{ Brassicaceae } \\
\hline $\begin{array}{l}\text { Erysimum } \\
\text { cheiranthoides L. }\end{array}$ & $\begin{array}{l}\text { Wormseed } \\
\text { wallflower }\end{array}$ & Chippewa & skin aid & root & decoction & $M$ \\
\hline \multirow{3}{*}{$\begin{array}{l}\text { Physaria } \\
\text { didymocarpa } \\
\text { (Hook.) A.Gray }\end{array}$} & \multirow[t]{3}{*}{$\begin{array}{l}\text { Common } \\
\text { twinpod }\end{array}$} & \multirow[t]{3}{*}{ Blackfeet } & skin aid & unknown & $\begin{array}{l}\text { infusion of plants } \\
\text { to heal wounds }\end{array}$ & $M$ \\
\hline & & & skin aid & unknown & $\begin{array}{l}\text { strong infusion } \\
\text { used as a liniment } \\
\text { for dislocations }\end{array}$ & $M$ \\
\hline & & & diaper rash & unknown & decoction & $M$ \\
\hline \multicolumn{7}{|l|}{ Fabaceae } \\
\hline $\begin{array}{l}\text { Astragalus } \\
\text { canadensis L. }\end{array}$ & $\begin{array}{l}\text { Canadian } \\
\text { milkvetch }\end{array}$ & Blackfeet & skin aid & root & $\begin{array}{l}\text { poultice of chewed } \\
\text { roots applied to cuts }\end{array}$ & $\mathrm{K} 2, \mathrm{M}$ \\
\hline
\end{tabular}




\begin{tabular}{|c|c|c|c|c|c|c|}
\hline \multicolumn{2}{|l|}{ Plant names } & \multirow{2}{*}{$\begin{array}{l}\text { Cultural } \\
\text { source }\end{array}$} & \multicolumn{3}{|c|}{ Plant information } & \multirow[t]{2}{*}{ Sources } \\
\hline Scientific & Common & & Uses & Parts used & Preparation & \\
\hline Dalea purpurea Vent. & $\begin{array}{l}\text { Purple prairie } \\
\text { clover }\end{array}$ & MT Indian & skin aid & leaves & $\begin{array}{l}\text { poultice of steeped } \\
\text { leaves applied to } \\
\text { fresh wounds }\end{array}$ & $\mathrm{B}, \mathrm{M}$ \\
\hline $\begin{array}{l}\text { Oxytropis lagopus } \\
\text { Nutt. }\end{array}$ & $\begin{array}{l}\text { Hare's-foot } \\
\text { locoweed }\end{array}$ & Blackfeet & skin aid & unknown & $\begin{array}{l}\text { plant chewed to } \\
\text { allay swelling }\end{array}$ & $M$ \\
\hline $\begin{array}{l}\text { Oxytropis sericea } \\
\text { Torr. \& A.Gray }\end{array}$ & $\begin{array}{l}\text { White } \\
\text { locoweed }\end{array}$ & Blackfeet & skin aid & leaves & infusion & $\mathrm{K} 2, \mathrm{M}$ \\
\hline $\begin{array}{l}\text { Psoralea argophylla } \\
\text { Pursh }\end{array}$ & $\begin{array}{l}\text { Silvery } \\
\text { scurfpea }\end{array}$ & MT Indian & skin aid & unknown & $\begin{array}{l}\text { decoction used for } \\
\text { washing wounds }\end{array}$ & $\mathrm{B}, \mathrm{M}$ \\
\hline $\begin{array}{l}\text { Psoralea esculenta } \\
\text { Pursh }\end{array}$ & $\begin{array}{l}\text { Pomme- } \\
\text { de-prairie }\end{array}$ & Cheyenne & $\begin{array}{l}\text { burn } \\
\text { dressing }\end{array}$ & unknown & unspecified & $\begin{array}{l}\mathrm{H} 1, \mathrm{H} 2 \\
\mathrm{~K} 1, \mathrm{~K} 2 \\
\mathrm{M}, \mathrm{T}\end{array}$ \\
\hline \multicolumn{7}{|l|}{ Lamiaceae } \\
\hline $\begin{array}{l}\text { Agastache } \\
\text { foeniculum (Pursh) } \\
\text { Kuntze }\end{array}$ & $\begin{array}{l}\text { Lavender } \\
\text { hyssop }\end{array}$ & Chippewa & $\begin{array}{l}\text { burn } \\
\text { dressing }\end{array}$ & $\begin{array}{l}\text { leaves } \\
\text { or stalk }\end{array}$ & $\begin{array}{l}\text { simple or compound } \\
\text { poultice }\end{array}$ & $M$ \\
\hline \multirow[t]{2}{*}{ Monarda fistulosa L. } & \multirow[t]{2}{*}{ Beebalm } & \multirow[t]{2}{*}{ Blackfeet } & skin aid & flowerhead & $\begin{array}{l}\text { poultice applied to } \\
\text { heal burst boils }\end{array}$ & $\mathrm{K} 2, \mathrm{M}$ \\
\hline & & & skin aid & unknown & $\begin{array}{l}\text { poultice applied } \\
\text { to heal cuts }\end{array}$ & $\mathrm{K} 2, \mathrm{M}$ \\
\hline Prunella vulgaris L. & Self-heal & Blackfeet & skin aid & unknown & $\begin{array}{l}\text { infusion applied to } \\
\text { wash a burst boil } \\
\text { or neck sore }\end{array}$ & $M$ \\
\hline \multicolumn{7}{|l|}{ Ranunculaceae } \\
\hline $\begin{array}{l}\text { Actaea rubra (Aiton) } \\
\text { Willd. }\end{array}$ & $\begin{array}{l}\text { Red } \\
\text { baneberry }\end{array}$ & Cheyenne & skin aid & root & unspecified & $\begin{array}{l}\mathrm{H} 1, \\
\mathrm{H} 2, \mathrm{M}\end{array}$ \\
\hline $\begin{array}{l}\text { Anemone canadensis } \\
\text { L. }\end{array}$ & $\begin{array}{l}\text { Canada } \\
\text { anemone }\end{array}$ & Chippewa & skin aid & root & poultice or infusion & $M$ \\
\hline
\end{tabular}

Table 9. Plants implied from the archaeological record at Beaver Creek Rock Shelter, southwestern Montana, U.S.A. with ethnographic uses as cough and cold remedies. Information sources: (G) Grinnell 1972, (H1) Hart 1981, (H2) Hart 1992, (J1) Johnston 1970, (J2) Johnston 1987, (K) Kindscher 1992, (M) Moerman 1998, and (S) Scheinost 2010.

\begin{tabular}{|c|c|c|c|c|c|c|}
\hline \multicolumn{2}{|l|}{ Plant names } & \multirow{2}{*}{$\begin{array}{l}\text { Cultural } \\
\text { source }\end{array}$} & \multicolumn{3}{|c|}{ Plant information } & \multirow[t]{2}{*}{ Sources } \\
\hline Scientific & Common & & Uses & Parts used & Preparation & \\
\hline \multicolumn{7}{|l|}{ Apiaceae } \\
\hline \multirow[t]{2}{*}{$\begin{array}{l}\text { Ligusticum canbyi } \\
\text { J.M. Coult. \& Rose }\end{array}$} & \multirow[t]{2}{*}{$\begin{array}{l}\text { Canby's wild } \\
\text { lovage }\end{array}$} & \multirow[t]{2}{*}{ Crow } & $\begin{array}{l}\text { cold } \\
\text { remedy }\end{array}$ & root & chewed & $\mathrm{H} 2, \mathrm{M}$ \\
\hline & & & $\begin{array}{l}\text { cough } \\
\text { medicine }\end{array}$ & root & chewed & $\mathrm{H} 2, \mathrm{M}$ \\
\hline $\begin{array}{l}\text { Peucedanum } \\
\text { macrocarpum Nutt. }\end{array}$ & $\begin{array}{l}\text { Large-fruit } \\
\text { desert-parsley }\end{array}$ & Crow & $\begin{array}{l}\text { cold } \\
\text { remedy }\end{array}$ & root & $\begin{array}{l}\text { infusion of shavings } \\
\text { and animal fat }\end{array}$ & $M$ \\
\hline \multirow[t]{2}{*}{$\begin{array}{l}\text { Osmorhiza chilensis } \\
\text { Hook. \& Arn. }\end{array}$} & \multirow[t]{2}{*}{$\begin{array}{l}\text { Chilean } \\
\text { sweet-cicely }\end{array}$} & Blackfeet & $\begin{array}{l}\text { cold } \\
\text { remedy }\end{array}$ & root & hot drink & $\mathrm{H} 1, \mathrm{M}$ \\
\hline & & Cheyenne & $\begin{array}{l}\text { cold } \\
\text { remedy }\end{array}$ & $\begin{array}{l}\text { root or } \\
\text { leaves }\end{array}$ & $\begin{array}{l}\text { chewed roots or } \\
\text { leaf infusion }\end{array}$ & $M$ \\
\hline $\begin{array}{l}\text { Osmorhiza } \\
\text { occidentalis } \\
\text { (Nutt.) Torr. }\end{array}$ & $\begin{array}{l}\text { Western } \\
\text { sweet-cicely }\end{array}$ & Blackfeet & $\begin{array}{l}\text { cough } \\
\text { medicine }\end{array}$ & unknown & infusion & $M$ \\
\hline
\end{tabular}


Dexter et al. - Prehistoric Plant Use at Beaver Creek Rock Shelter,

Southwestern Montana, U.S.A.

\begin{tabular}{|c|c|c|c|c|c|c|}
\hline \multicolumn{2}{|l|}{ Plant names } & \multirow{2}{*}{$\begin{array}{l}\text { Cultural } \\
\text { source }\end{array}$} & \multicolumn{3}{|c|}{ Plant information } & \multirow[t]{2}{*}{ Sources } \\
\hline Scientific & Common & & Uses & Parts used & Preparation & \\
\hline $\begin{array}{l}\text { Perideridia montana } \\
\text { (Blank.) Dorn }\end{array}$ & $\begin{array}{l}\text { Gairdner's } \\
\text { yampah }\end{array}$ & Blackfeet & $\begin{array}{l}\text { cough } \\
\text { medicine }\end{array}$ & root & $\begin{array}{l}\text { infusion, chewed or } \\
\text { smudge smoke for } \\
\text { nagging cough }\end{array}$ & $M$ \\
\hline \multicolumn{7}{|l|}{ Brassicaceae } \\
\hline Turritis glabra L. & $\begin{array}{l}\text { Tower- } \\
\text { mustard }\end{array}$ & Cheyenne & $\begin{array}{l}\text { cold } \\
\text { remedy }\end{array}$ & unknown & infusion & G, M \\
\hline \multicolumn{7}{|l|}{ Fabaceae } \\
\hline $\begin{array}{l}\text { Glycyrrhiza lepidota } \\
\text { Pursh }\end{array}$ & Wild licorice & Blackfeet & $\begin{array}{l}\text { cough } \\
\text { medicine }\end{array}$ & root & infusion & $M$ \\
\hline $\begin{array}{l}\text { Thermopsis } \\
\text { rhombifolia (Pursh) } \\
\text { Richardson }\end{array}$ & $\begin{array}{l}\text { Roundleaf } \\
\text { thermopsis }\end{array}$ & Cheyenne & $\begin{array}{l}\text { cold } \\
\text { remedy }\end{array}$ & leaves & $\begin{array}{l}\text { dried, burned, } \\
\text { and inhaled }\end{array}$ & $\mathrm{H} 1, \mathrm{M}$ \\
\hline \multicolumn{7}{|l|}{ Geraniaceae } \\
\hline $\begin{array}{l}\text { Geranium } \\
\text { viscosissimum } \\
\text { Fisch. \& C.A.Mey. }\end{array}$ & $\begin{array}{l}\text { Sticky } \\
\text { geranium }\end{array}$ & Blackfeet & $\begin{array}{l}\text { cold } \\
\text { remedy }\end{array}$ & leaves & $\begin{array}{l}\text { infusion with a } \\
\text { sweat bath }\end{array}$ & $M, S$ \\
\hline \multicolumn{7}{|l|}{ Lamiaceae } \\
\hline \multirow{2}{*}{$\begin{array}{l}\text { Agastache } \\
\text { foeniculum } \\
\text { (Pursh) Kuntze }\end{array}$} & \multirow[t]{2}{*}{$\begin{array}{l}\text { Lavender } \\
\text { hyssop }\end{array}$} & Cheyenne & $\begin{array}{l}\text { cold } \\
\text { remedy }\end{array}$ & leaves & infusion & $\mathrm{K}, \mathrm{M}$ \\
\hline & & Chippewa & $\begin{array}{l}\text { cough } \\
\text { medicine }\end{array}$ & root & infusion & $\mathrm{K}, \mathrm{M}$ \\
\hline \multirow[t]{5}{*}{ Mentha arvensis $\mathrm{L}$. } & \multirow[t]{5}{*}{ Wild mint } & Cree & $\begin{array}{l}\text { cold } \\
\text { remedy }\end{array}$ & leaves & infusion & $\mathrm{C}, \mathrm{H} 2, \mathrm{M}$ \\
\hline & & \multirow[t]{2}{*}{ Salish } & $\begin{array}{l}\text { cold } \\
\text { remedy }\end{array}$ & leaves & infusion & $\mathrm{C}, \mathrm{H} 2, \mathrm{M}$ \\
\hline & & & $\begin{array}{l}\text { cough } \\
\text { medicine }\end{array}$ & leaves & infusion & $\mathrm{C}, \mathrm{H} 2, \mathrm{M}$ \\
\hline & & \multirow[t]{2}{*}{ Kutenai } & $\begin{array}{l}\text { cough } \\
\text { medicine }\end{array}$ & unknown & infusion & $\mathrm{C}, \mathrm{H} 2, \mathrm{M}$ \\
\hline & & & $\begin{array}{l}\text { cold } \\
\text { remedy }\end{array}$ & unknown & infusion & $\mathrm{C}, \mathrm{H} 2, \mathrm{M}$ \\
\hline \multirow[t]{5}{*}{ Monarda fistulosa L. } & \multirow[t]{5}{*}{ Beebalm } & Blackfeet & $\begin{array}{l}\text { cough } \\
\text { medicine }\end{array}$ & unknown & infusion & $\mathrm{K}, \mathrm{M}$ \\
\hline & & Chippewa & $\begin{array}{l}\text { cold } \\
\text { remedy }\end{array}$ & plant tops & unspecified & $\mathrm{K}, \mathrm{M}$ \\
\hline & & \multirow[t]{3}{*}{ Salish } & $\begin{array}{l}\text { cold } \\
\text { remedy }\end{array}$ & unknown & infusion & $\mathrm{K}, \mathrm{M}$ \\
\hline & & & $\begin{array}{l}\text { cold } \\
\text { remedy }\end{array}$ & unknown & hung on the wall & $\mathrm{K}, \mathrm{M}$ \\
\hline & & & $\begin{array}{l}\text { cough } \\
\text { medicine }\end{array}$ & unknown & unspecified & $\mathrm{K}, \mathrm{M}$ \\
\hline \multicolumn{7}{|l|}{ Ranunculaceae } \\
\hline $\begin{array}{l}\text { Actaea rubra (Aiton) } \\
\text { Willd. }\end{array}$ & $\begin{array}{l}\text { Red } \\
\text { baneberry }\end{array}$ & Blackfeet & $\begin{array}{l}\text { cold } \\
\text { remedy }\end{array}$ & root & decoction & $\mathrm{J} 1, \mathrm{~J} 2, \mathrm{M}$ \\
\hline
\end{tabular}




\begin{tabular}{|c|c|c|c|c|c|c|}
\hline \multicolumn{2}{|l|}{ Plant names } & \multirow{2}{*}{\begin{tabular}{|l} 
Cultural \\
source
\end{tabular}} & \multicolumn{3}{|c|}{ Plant information } & \multirow[t]{2}{*}{ Sources } \\
\hline Scientific & Common & & Uses & Parts used & Preparation & \\
\hline \multirow[t]{2}{*}{$\begin{array}{l}\text { Clematis ligusticifolia } \\
\text { Nutt. }\end{array}$} & \multirow[t]{2}{*}{$\begin{array}{l}\text { Western } \\
\text { virgin's-bower }\end{array}$} & MT Indian & $\begin{array}{l}\text { cold } \\
\text { remedy }\end{array}$ & unknown & chewed & $\mathrm{J} 1, \mathrm{~J} 2, \mathrm{M}$ \\
\hline & & Blackfeet & $\begin{array}{l}\text { cold } \\
\text { remedy }\end{array}$ & foliage & unspecified & $\mathrm{J} 1, \mathrm{~J} 2, \mathrm{M}$ \\
\hline
\end{tabular}

ily with leaves, were the most frequently used method of preparation.

Medicinal remedies for heart, lung, and kidney ailments

Species in 4 of the 7 plant families were used as medicinal remedies for heart, lung, and kidney ailments, Apiaceae and Lamiaceae having the largest variety of different plant species with 4 each (Table 10). Lung disorders are labeled as pulmonary or respiratory aids and are the most frequently listed. The favored preparation technique was infusions made from roots.

\section{Traditional antihemorrhage and hemostat use}

Species in all 7 plant families were traditionally used for preparing hemostatic and antihemorrhagic medicines (Ta- ble 11). When controlling bleeding, root infusions were the most commonly used.

\section{$\underline{\text { Traditional medicinal feminine care }}$}

Species in 4 of the 7 plant families were used in traditional medicinal feminine care. Uses consist of birthing aids, abortifacients, breast and breastfeeding aids, a menstrual aid, a feminine deodorant, a general gynecological remedy, and a newborn aid (Table 12). Prepared root infusions are the primary form of treatment.

Ethnographic hunting aids

Ethnographic hunting aids include animal attractants, athletic strengtheners, a water-proofing agent, and a thirst aid and were found in 4 of the 7 plant families (Table 13). Notably, Cicuta maculata L. and R. pensylvanicus-both deer attractants from different plant families-use differ-

Table 10. Plants implied from the archaeological record at Beaver Creek Rock Shelter, southwestern Montana, U.S.A. with ethnographic uses as remedies for heart, lung, and kidney ailments. Information sources: (H1) Hart 1981, (H2) Hart 1992, (H3) Hoffman 1891, (K1) Kindscher 1987, (K2) Kindscher 1992, (M) Moerman 1998, and (T) Taylor 1989.

\begin{tabular}{|c|c|c|c|c|c|c|}
\hline \multicolumn{2}{|l|}{ Plant names } & \multirow{2}{*}{$\begin{array}{l}\text { Cultural } \\
\text { source }\end{array}$} & \multicolumn{3}{|c|}{ Plant information } & \multirow[t]{2}{*}{ Sources } \\
\hline Scientific & Common & & Uses & Parts used & Preparation & \\
\hline \multicolumn{7}{|l|}{ Apiaceae } \\
\hline \multirow{2}{*}{$\begin{array}{l}\text { Ligusticum canbyi } \\
\text { J.M. Coult. \& Rose }\end{array}$} & \multirow{2}{*}{$\begin{array}{l}\text { Canby's wild } \\
\text { lovage }\end{array}$} & Cree & heart aid & root & unspecified & $\mathrm{H} 2, \mathrm{M}$ \\
\hline & & Crow & $\begin{array}{l}\text { respiratory } \\
\text { aid }\end{array}$ & root & $\begin{array}{l}\text { shavings added } \\
\text { to boiling water to } \\
\text { inhale steam }\end{array}$ & $\mathrm{H} 2, \mathrm{M}$ \\
\hline $\begin{array}{l}\text { Lomatium triternatum } \\
\text { (Pursh) J.M.Coult. } \\
\text { \& Rose }\end{array}$ & $\begin{array}{l}\text { Nineleaf } \\
\text { biscuitroot }\end{array}$ & Blackfeet & $\begin{array}{l}\text { pulmonary } \\
\text { aid }\end{array}$ & $\begin{array}{l}\text { roots and } \\
\text { leaves }\end{array}$ & infusion & $M$ \\
\hline $\begin{array}{l}\text { Osmorhiza longistylis } \\
\text { (Torr.) DC. }\end{array}$ & $\begin{array}{l}\text { Smoother } \\
\text { sweet-cicely }\end{array}$ & Cheyenne & kidney aid & $\begin{array}{l}\text { leaves, } \\
\text { stem, root }\end{array}$ & infusion & $M$ \\
\hline $\begin{array}{l}\text { Perideridia montana } \\
\text { (Blank.) Dorn }\end{array}$ & $\begin{array}{l}\text { Gairdner's } \\
\text { yampah }\end{array}$ & Blackfeet & $\begin{array}{l}\text { respiratory } \\
\text { aid }\end{array}$ & root & infusion & $M$ \\
\hline \multicolumn{7}{|l|}{ Fabacaeae } \\
\hline Dalea purpurea Vent. & $\begin{array}{l}\text { Purple prairie } \\
\text { clover }\end{array}$ & Chippewa & heart aid & $\begin{array}{l}\text { leaves and } \\
\text { blossoms }\end{array}$ & decoction & $M$ \\
\hline $\begin{array}{l}\text { Oxytropis lagopus } \\
\text { Nutt. }\end{array}$ & $\begin{array}{l}\text { Hare's-foot } \\
\text { locoweed }\end{array}$ & Blackfeet & $\begin{array}{l}\text { respiratory } \\
\text { aid }\end{array}$ & unknown & eaten for asthma & K2 \\
\hline $\begin{array}{l}\text { Psoralea esculenta } \\
\text { Pursh }\end{array}$ & $\begin{array}{l}\text { Pomme- } \\
\text { de-prairie }\end{array}$ & Blackfeet & $\begin{array}{l}\text { pulmonary } \\
\text { aid }\end{array}$ & root & chewed & $\begin{array}{l}\mathrm{H} 2, \mathrm{~K} 1 \\
\mathrm{~K} 2, \mathrm{M}, \mathrm{T}\end{array}$ \\
\hline
\end{tabular}


Dexter et al. - Prehistoric Plant Use at Beaver Creek Rock Shelter,

Southwestern Montana, U.S.A.

\begin{tabular}{|c|c|c|c|c|c|c|}
\hline \multicolumn{2}{|l|}{ Plant names } & \multirow{2}{*}{$\begin{array}{l}\text { Cultural } \\
\text { source }\end{array}$} & \multicolumn{3}{|c|}{ Plant information } & \multirow[t]{2}{*}{ Sources } \\
\hline Scientific & Common & & Uses & Parts used & Preparation & \\
\hline \multicolumn{7}{|l|}{ Lamiaceae } \\
\hline $\begin{array}{l}\text { Agastache } \\
\text { foeniculum (Pursh) } \\
\text { Kuntze }\end{array}$ & $\begin{array}{l}\text { Lavender } \\
\text { hyssop }\end{array}$ & Cheyenne & heart aid & leaves & $\begin{array}{l}\text { infusion for a } \\
\text { weak heart }\end{array}$ & $\mathrm{K} 2, \mathrm{M}$ \\
\hline \multirow[t]{3}{*}{ Mentha arvensis L. } & \multirow[t]{3}{*}{ Wild mint } & Cheyenne & heart aid & $\begin{array}{l}\text { leaves } \\
\text { and stem }\end{array}$ & $\begin{array}{l}\text { ground infusion or } \\
\text { boiled to strengthen } \\
\text { the heart }\end{array}$ & $\begin{array}{l}\mathrm{H} 1, \mathrm{H} 2 \\
\mathrm{~K} 2, \mathrm{M}\end{array}$ \\
\hline & & Kutenai & kidney aid & unknown & infusion & $\begin{array}{l}\mathrm{H} 1, \mathrm{H} 2 \\
\mathrm{~K} 2, \mathrm{M}\end{array}$ \\
\hline & & Blackfeet & heart aid & leaves & unspecified & $\begin{array}{l}\mathrm{H} 1, \mathrm{H} 2 \\
\mathrm{~K} 2, \mathrm{M}\end{array}$ \\
\hline \multirow[t]{4}{*}{ Monarda fistulosa $\mathrm{L}$. } & \multirow[t]{4}{*}{ Beebalm } & Blackfeet & kidney aid & unknown & infusion & $\mathrm{K} 2, \mathrm{M}$ \\
\hline & & Salish & $\begin{array}{l}\text { pulmonary } \\
\text { aid }\end{array}$ & unknown & infusion for pneumonia & $\mathrm{K} 2, \mathrm{M}$ \\
\hline & & Kutenai & kidney aid & unknown & infusion & $\mathrm{K} 2, \mathrm{M}$ \\
\hline & & Ojibwa & $\begin{array}{l}\text { respiratory } \\
\text { aid }\end{array}$ & unknown & $\begin{array}{l}\text { boiled and inhale } \\
\text { the steam }\end{array}$ & $\mathrm{H} 3, \mathrm{M}$ \\
\hline $\begin{array}{l}\text { Scutellaria } \\
\text { galericulata L. }\end{array}$ & $\begin{array}{l}\text { Hooded } \\
\text { skullcap }\end{array}$ & Ojibwa & heart aid & unknown & unspecified & $M, S$ \\
\hline \multicolumn{7}{|l|}{ Ranunculaceae } \\
\hline $\begin{array}{l}\text { Anemone cylindrica } \\
\text { A.Gray }\end{array}$ & $\begin{array}{l}\text { Long-fruited } \\
\text { anemone }\end{array}$ & Ojibwa & $\begin{array}{l}\text { pulmonary } \\
\text { aid }\end{array}$ & root & infusion & $M, S$ \\
\hline $\begin{array}{l}\text { Thalictrum } \\
\text { occidentale A.Gray }\end{array}$ & $\begin{array}{l}\text { Western } \\
\text { meadowrue }\end{array}$ & Blackfeet & $\begin{array}{l}\text { pulmonary } \\
\text { aid }\end{array}$ & seeds & infusion & $M$ \\
\hline
\end{tabular}

Table 11. Plants implied from the archaeological record at Beaver Creek Rock Shelter, southwestern Montana, U.S.A. with ethnographic antihemorrhage and hemostat uses. Information sources: (D) Densmore 1927, (H) Hart 1981, (K) Kindscher 1992, (M) Moerman 1998, (R) Reaves 2005, and (S) Smith 1932.

\begin{tabular}{|c|c|c|c|c|c|c|}
\hline \multicolumn{2}{|l|}{ Plant names } & \multirow{2}{*}{$\begin{array}{l}\text { Cultural } \\
\text { source }\end{array}$} & \multicolumn{3}{|c|}{ Plant information } & \multirow[t]{2}{*}{ Sources } \\
\hline Scientific & Common & & Uses & Parts used & Preparation & \\
\hline \multicolumn{7}{|l|}{ Apiaceae } \\
\hline $\begin{array}{l}\text { Angelica dawsonii } \\
\text { S.Watson }\end{array}$ & $\begin{array}{l}\text { Dawson's } \\
\text { angelica }\end{array}$ & Blackfeet & hemostat & root & infusion & $M$ \\
\hline \multicolumn{7}{|l|}{ Brassicaceae } \\
\hline Draba incerta Payson & $\begin{array}{l}\text { Yellowstone } \\
\text { whitlow-grass }\end{array}$ & Blackfeet & hemostat & root & $\begin{array}{l}\text { infusion used to } \\
\text { stop nose bleeds }\end{array}$ & M \\
\hline \multicolumn{7}{|l|}{ Cyperaceae } \\
\hline $\begin{array}{l}\text { Eriophorum callitrix } \\
\text { Cham. ex C.A.Mey. }\end{array}$ & $\begin{array}{l}\text { Sheathed } \\
\text { cotton-grass }\end{array}$ & Ojibwa & hemostat & matted fuzz & unspecified & $M, S$ \\
\hline $\begin{array}{l}\text { Schoenoplectus } \\
\text { acutus (Muhl. ex } \\
\text { Bigelow) Á.Löve } \\
\text { \& D.Löve }\end{array}$ & $\begin{array}{l}\text { Hardstem } \\
\text { bulrush }\end{array}$ & Cree & hemostat & stem & $\begin{array}{l}\text { poultice of stem } \\
\text { pith applied under } \\
\text { a dressing }\end{array}$ & $\mathrm{M}$ \\
\hline \multicolumn{7}{|l|}{ Fabaceae } \\
\hline $\begin{array}{l}\text { Astragalus } \\
\text { canadensis L. }\end{array}$ & $\begin{array}{l}\text { Canadian } \\
\text { milkvetch }\end{array}$ & Blackfeet & $\begin{array}{l}\text { antihemor- } \\
\text { rhagic }\end{array}$ & root & $\begin{array}{l}\text { chewed when } \\
\text { spitting blood }\end{array}$ & $\mathrm{K}, \mathrm{M}$ \\
\hline
\end{tabular}




\begin{tabular}{|c|c|c|c|c|c|c|}
\hline \multicolumn{2}{|l|}{ Plant names } & \multirow{2}{*}{$\begin{array}{l}\text { Cultural } \\
\text { source }\end{array}$} & \multicolumn{3}{|c|}{ Plant information } & \multirow[t]{2}{*}{ Sources } \\
\hline Scientific & Common & & Uses & Parts used & Preparation & \\
\hline $\begin{array}{l}\text { Astragalus } \\
\text { crassicarpus Nutt. }\end{array}$ & $\begin{array}{l}\text { Groundplum } \\
\text { milkvetch }\end{array}$ & Chippewa & hemostat & root & decoction & $D, M$ \\
\hline \multicolumn{7}{|l|}{ Geraniaceae } \\
\hline $\begin{array}{l}\text { Geranium } \\
\text { richardsonii } \\
\text { Fisch. \& Trautv. }\end{array}$ & $\begin{array}{l}\text { Richardson's } \\
\text { geranium }\end{array}$ & Cheyenne & hemostat & $\begin{array}{l}\text { roots or } \\
\text { leaves }\end{array}$ & $\begin{array}{l}\text { infusion of dried root } \\
\text { or powdered leaves } \\
\text { used as a snuff to } \\
\text { stop nose bleeds }\end{array}$ & $\mathrm{H}, \mathrm{M}, \mathrm{R}$ \\
\hline \multicolumn{7}{|l|}{ Lamiaceae } \\
\hline $\begin{array}{l}\text { Agastache } \\
\text { foeniculum } \\
\text { (Pursh) Kuntze }\end{array}$ & $\begin{array}{l}\text { Lavender } \\
\text { hyssop }\end{array}$ & Cree & $\begin{array}{l}\text { antihemor- } \\
\text { rhagic }\end{array}$ & $\begin{array}{l}\text { leaves and } \\
\text { stems }\end{array}$ & $\begin{array}{l}\text { infusion for } \\
\text { spitting blood }\end{array}$ & $\mathrm{D}, \mathrm{M}$ \\
\hline \multirow[t]{2}{*}{ Mentha arvensis L. } & \multirow[t]{2}{*}{ Wild mint } & Cree & $\begin{array}{l}\text { antihemor- } \\
\text { rhagic } \\
\end{array}$ & unknown & plant infusion & $M$ \\
\hline & & Cree & hemostat & $\begin{array}{l}\text { leafy stems } \\
\text { and flowers }\end{array}$ & $\begin{array}{l}\text { inserted into the nose } \\
\text { to stop bleeding }\end{array}$ & $M$ \\
\hline \multicolumn{7}{|l|}{ Ranunculaceae } \\
\hline $\begin{array}{l}\text { Anemone canadensis } \\
\text { L. }\end{array}$ & $\begin{array}{l}\text { Canada } \\
\text { anemone }\end{array}$ & Chippewa & hemostat & leaves & $\begin{array}{l}\text { used to stop } \\
\text { bleeding noses, } \\
\text { sores and wounds }\end{array}$ & $M$ \\
\hline
\end{tabular}

Table 12. Plants implied from the archaeological record at Beaver Creek Rock Shelter, southwestern Montana, U.S.A. with ethnographic medicinal feminine care uses. Information sources: (C) Clavelle 1997, (H1) Hart 1981, (H2) Holmes 1884, (K) Kindscher 1992, (M) Moerman 1998, (S) Smith 1932, (T1) Taylor 1989, and (T2) Turner 1971.

\begin{tabular}{|c|c|c|c|c|c|c|}
\hline \multicolumn{2}{|l|}{ Plant names } & \multirow{2}{*}{$\begin{array}{l}\text { Cultural } \\
\text { source }\end{array}$} & \multicolumn{3}{|c|}{ Plant information } & \multirow[t]{2}{*}{ Sources } \\
\hline Scientific & Common & & Uses & Parts used & Preparation & \\
\hline \multicolumn{7}{|l|}{ Apiaceae } \\
\hline \multirow[t]{2}{*}{$\begin{array}{l}\text { Osmorhiza longistylis } \\
\text { (Torr.) DC. }\end{array}$} & \multirow[t]{2}{*}{$\begin{array}{l}\text { Smoother } \\
\text { sweet-cicely }\end{array}$} & Chippewa & $\begin{array}{l}\text { menstrual } \\
\text { aid }\end{array}$ & root & $\begin{array}{l}\text { infusion to treat } \\
\text { amenorrhea }\end{array}$ & $M$ \\
\hline & & Ojibwa & birthing aid & root & infusion & $\mathrm{M}, \mathrm{S}$ \\
\hline \multirow{3}{*}{$\begin{array}{l}\text { Osmorhiza } \\
\text { occidentalis } \\
\text { (Nutt.) Torr. }\end{array}$} & \multirow[t]{3}{*}{$\begin{array}{l}\text { Western } \\
\text { sweet-cicely }\end{array}$} & \multirow[t]{3}{*}{ Blackfeet } & breast aid & root & $\begin{array}{l}\text { infusion to treat } \\
\text { swollen breasts }\end{array}$ & $M$ \\
\hline & & & $\begin{array}{l}\text { feminine } \\
\text { deodorant }\end{array}$ & root & infusion & $M$ \\
\hline & & & birthing aid & root & $\begin{array}{l}\text { infusion to } \\
\text { induce labor }\end{array}$ & $M$ \\
\hline $\begin{array}{l}\text { Perideridia montana } \\
\text { (Blank.) Dorn }\end{array}$ & $\begin{array}{l}\text { Gairdner's } \\
\text { yampah }\end{array}$ & Blackfeet & breast aid & root & $\begin{array}{l}\text { infusion used to } \\
\text { massage sore breasts } \\
\text { with warm stones }\end{array}$ & $M$ \\
\hline \multicolumn{7}{|l|}{ Brassicaceae } \\
\hline Draba incerta Payson & $\begin{array}{l}\text { Yellowstone } \\
\text { whitlow-grass }\end{array}$ & Blackfeet & $\begin{array}{l}\text { aborti- } \\
\text { facient }\end{array}$ & unknown & unspecified & $M$ \\
\hline \multirow[t]{2}{*}{$\begin{array}{l}\text { Physaria } \\
\text { didymocarpa } \\
\text { (Hook.) A.Gray }\end{array}$} & \multirow[t]{2}{*}{$\begin{array}{l}\text { Common } \\
\text { twinpod }\end{array}$} & \multirow[t]{2}{*}{ Blackfeet } & $\begin{array}{l}\text { newborn } \\
\text { aid }\end{array}$ & leaves & $\begin{array}{l}\text { decoction to aid in } \\
\text { the healing of the } \\
\text { umbilical cord }\end{array}$ & $M$ \\
\hline & & & $\begin{array}{l}\text { aborti- } \\
\text { facient }\end{array}$ & unknown & $\begin{array}{l}\text { infusion taken in } \\
\text { small amounts }\end{array}$ & $M$ \\
\hline
\end{tabular}


Dexter et al. - Prehistoric Plant Use at Beaver Creek Rock Shelter,

Southwestern Montana, U.S.A.

\begin{tabular}{|c|c|c|c|c|c|c|}
\hline \multicolumn{2}{|l|}{ Plant names } & \multirow{2}{*}{$\begin{array}{l}\text { Cultural } \\
\text { source }\end{array}$} & \multicolumn{3}{|c|}{ Plant information } & \multirow[t]{2}{*}{ Sources } \\
\hline Scientific & Common & & Uses & Parts used & Preparation & \\
\hline \multicolumn{7}{|l|}{ Lamiaceae } \\
\hline \multirow[t]{2}{*}{ Monarda fistulosa L. } & \multirow[t]{2}{*}{ Beebalm } & Salish & birthing aid & unknown & $\begin{array}{l}\text { beverage drunk } \\
\text { after childbirth }\end{array}$ & $\mathrm{K}, \mathrm{M}$ \\
\hline & & MT Indian & birthing aid & unknown & $\begin{array}{l}\text { infusion to aid in the } \\
\text { expulsion of afterbirth }\end{array}$ & $\mathrm{K}, \mathrm{M}$ \\
\hline Prunella vulgaris $\mathrm{L}$. & Self-heal & Ojibwa & $\begin{array}{l}\text { gyne- } \\
\text { cological } \\
\text { remedy }\end{array}$ & root & compound & $M, S$ \\
\hline \multicolumn{7}{|l|}{ Ranunculaceae } \\
\hline \multirow[t]{6}{*}{$\begin{array}{l}\text { Actaea rubra } \\
\text { (Aiton) Willd. }\end{array}$} & \multirow[t]{6}{*}{$\begin{array}{l}\text { Red } \\
\text { baneberry }\end{array}$} & \multirow[t]{2}{*}{ Cheyenne } & \multirow[t]{2}{*}{$\begin{array}{l}\text { breast } \\
\text { feeding aid }\end{array}$} & root & $\begin{array}{l}\text { infusion used after } \\
\text { childbirth to increase } \\
\text { the milk flow }\end{array}$ & $M$ \\
\hline & & & & stem & $\begin{array}{l}\text { infusion taken } \\
\text { by pregnant or } \\
\text { breastfeeding } \\
\text { mothers to increase } \\
\text { the milk flow }\end{array}$ & $\mathrm{H} 1, \mathrm{M}$ \\
\hline & & Chippewa & $\begin{array}{l}\text { menstrual } \\
\text { aid }\end{array}$ & root & $\begin{array}{l}\text { decoction to } \\
\text { treat excessive } \\
\text { menstrual flow }\end{array}$ & $M$ \\
\hline & & \multirow[t]{2}{*}{ Cree } & $\begin{array}{l}\text { menstrual } \\
\text { aid }\end{array}$ & root & $\begin{array}{l}\text { infusion to } \\
\text { treat excessive } \\
\text { menstrual flow }\end{array}$ & $\mathrm{C}, \mathrm{H} 2, \mathrm{M}$ \\
\hline & & & birthing aid & whole plant & $\begin{array}{l}\text { infusion taken to } \\
\text { clear up the birthing } \\
\text { system after childbirth }\end{array}$ & $\mathrm{C}, \mathrm{M}$ \\
\hline & & Ojibwa & birthing aid & root & $\begin{array}{l}\text { infusion taken to } \\
\text { clear up the birthing } \\
\text { system after childbirth }\end{array}$ & $M$ \\
\hline $\begin{array}{l}\text { Anemone multifida } \\
\text { Poir. }\end{array}$ & $\begin{array}{l}\text { Pacific } \\
\text { anemone }\end{array}$ & Blackfeet & $\begin{array}{l}\text { aborti- } \\
\text { facient }\end{array}$ & unknown & unspecified & $\mathrm{K}, \mathrm{M}$ \\
\hline
\end{tabular}

Table 13. Plants implied from the archaeological record at Beaver Creek Rock Shelter, southwestern Montana, U.S.A. with ethnographic uses as hunting aids. Information sources: (G) Gilmore 1977, (J) Johnston 1970, (M) Moerman 1998, and (S) Smith 1932.

\begin{tabular}{|c|c|c|c|c|c|c|}
\hline \multicolumn{2}{|l|}{ Plant names } & \multirow{2}{*}{$\begin{array}{l}\text { Cultural } \\
\text { source }\end{array}$} & \multicolumn{3}{|c|}{ Plant information } & \multirow[t]{2}{*}{ Sources } \\
\hline Scientific & Common & & Uses & Parts used & Preparation & \\
\hline \multicolumn{7}{|l|}{ Apiaceae } \\
\hline Cicuta maculata L. & $\begin{array}{l}\text { Spotted water- } \\
\text { hemlock }\end{array}$ & Ojibwa & $\begin{array}{l}\text { animal } \\
\text { attractant }\end{array}$ & root & $\begin{array}{l}\text { smoke of burning root } \\
\text { used to bring buck } \\
\text { deer close enough to } \\
\text { shoot with an arrow }\end{array}$ & $M, S$ \\
\hline \multirow[t]{2}{*}{$\begin{array}{l}\text { Perideridia montana } \\
\text { (Blank.) Dorn }\end{array}$} & \multirow[t]{2}{*}{$\begin{array}{l}\text { Gairdner's } \\
\text { yampah }\end{array}$} & \multirow[t]{2}{*}{ Blackfeet } & $\begin{array}{l}\text { athletic } \\
\text { strength }\end{array}$ & root & $\begin{array}{l}\text { chewed to give buffalo } \\
\text { hunter endurance }\end{array}$ & M \\
\hline & & & hunting aid & whole plant & $\begin{array}{l}\text { rubbed on arrows as } \\
\text { a waterproofing agent }\end{array}$ & M \\
\hline
\end{tabular}




\begin{tabular}{|c|c|c|c|c|c|c|}
\hline \multicolumn{2}{|l|}{ Plant names } & \multirow{2}{*}{$\begin{array}{l}\text { Cultural } \\
\text { source }\end{array}$} & \multicolumn{3}{|c|}{ Plant information } & \multirow[t]{2}{*}{ Sources } \\
\hline Scientific & Common & & Uses & Parts used & Preparation & \\
\hline \multicolumn{7}{|l|}{ Fabacaceae } \\
\hline $\begin{array}{l}\text { Amorpha canescens } \\
\text { Pursh }\end{array}$ & Lead plant & MT Indian & $\begin{array}{l}\text { animal } \\
\text { attractant }\end{array}$ & blooms & $\begin{array}{l}\text { pound, moisten, mix, } \\
\text { and rub on clothing }\end{array}$ & G \\
\hline $\begin{array}{l}\text { Glycyrrhiza lepidota } \\
\text { Pursh }\end{array}$ & Wild licorice & Blackfeet & thirst aid & burs & $\begin{array}{l}\text { kept in mouth of } \\
\text { buffalo runners to } \\
\text { prevent thirst }\end{array}$ & $M$ \\
\hline \multicolumn{7}{|l|}{ Lamiaceae } \\
\hline Mentha arvensis L. & Wild mint & Blackfeet & $\begin{array}{l}\text { animal } \\
\text { attractant }\end{array}$ & unknown & $\begin{array}{l}\text { boiled with hunting } \\
\text { traps to destroy } \\
\text { human scent }\end{array}$ & $\mathrm{J}$ \\
\hline \multicolumn{7}{|l|}{ Ranunculaceae } \\
\hline $\begin{array}{l}\text { Ranunculus } \\
\text { pensylvanicus L.f. }\end{array}$ & $\begin{array}{l}\text { Bristly } \\
\text { crowfoot }\end{array}$ & Ojibwa & $\begin{array}{l}\text { animal } \\
\text { attractant }\end{array}$ & seeds & $\begin{array}{l}\text { burned to lure buck } \\
\text { deer close enough to } \\
\text { shoot with an arrow }\end{array}$ & $\mathrm{M}, \mathrm{S}$ \\
\hline
\end{tabular}

ent plant parts, yet the preparation of the plants was identical.

\section{Ethnographic stimulants}

Ethnographic stimulants consist of 4 stimulants and 2 strengtheners with the majority of species from the Apiaceae family (Table 14). The plant part most commonly used was roots, which were prepared in a variety of ways including chewing, infusions, a tonic, and a decoction.

\section{Additional medicinal aids}

Additional medicinal aids are remedies that did not fit into any other categories, including plants that were used for panacea, snakebites, diaphoretics, a blood remedy, and an orthopedic aid. Ten species of plants used as additional medicinal aids occur in 4 of the 7 plant families (Table 15). Infusions made from roots were the most common treatment for these ailments.

Table 14. Plants implied from the archaeological record at Beaver Creek Rock Shelter, southwestern Montana, U.S.A. with ethnographic uses as stimulents. Information sources: (D) Densmore 1927, (K) Kindscher 1992, and (M) Moerman 1998.

\begin{tabular}{|c|c|c|c|c|c|c|}
\hline \multicolumn{2}{|l|}{ Plant names } & \multirow{2}{*}{$\begin{array}{l}\text { Cultural } \\
\text { source }\end{array}$} & \multicolumn{3}{|c|}{ Plant information } & \multirow[t]{2}{*}{ Sources } \\
\hline Scientific & Common & & Uses & Parts used & Preparation & \\
\hline \multicolumn{7}{|l|}{ Apiaceae } \\
\hline $\begin{array}{l}\text { Lomatium triternatum } \\
\text { (Pursh) J.M.Coult. } \\
\text { \& Rose }\end{array}$ & $\begin{array}{l}\text { Nineleaf } \\
\text { biscuitroot }\end{array}$ & Blackfeet & $\begin{array}{l}\text { athletic } \\
\text { strength }\end{array}$ & root & $\begin{array}{l}\text { chewed to prevent } \\
\text { side aches while } \\
\text { running }\end{array}$ & $M$ \\
\hline $\begin{array}{l}\text { Musineon divaricatum } \\
\text { (Pursh) Nutt. }\end{array}$ & Wild parsley & Blackfeet & stimulant & root & $\begin{array}{l}\text { tonic to stimulate } \\
\text { the appetite }\end{array}$ & $\mathrm{K}, \mathrm{M}$ \\
\hline $\begin{array}{l}\text { Osmorhiza chilensis } \\
\text { Hook. \& Arn. }\end{array}$ & $\begin{array}{l}\text { Chilean } \\
\text { sweet-cicely }\end{array}$ & Cheyenne & stimulant & $\begin{array}{l}\text { root or } \\
\text { leaves }\end{array}$ & $\begin{array}{l}\text { chewed roots or } \\
\text { infusion of leaves to } \\
\text { bring one around }\end{array}$ & $M$ \\
\hline $\begin{array}{l}\text { Peucedanum } \\
\text { macrocarpum Nutt. }\end{array}$ & $\begin{array}{l}\text { Large-fruit } \\
\text { desert-parsley }\end{array}$ & Blackfeet & strength & root & infusion & $M$ \\
\hline \multicolumn{7}{|l|}{ Fabaceae } \\
\hline $\begin{array}{l}\text { Astragalus } \\
\text { crassicarpus Nutt. }\end{array}$ & $\begin{array}{l}\text { Groundplum } \\
\text { milkvetch }\end{array}$ & Chippewa & stimulant & root & decoction & $\mathrm{D}, \mathrm{M}$ \\
\hline \multicolumn{7}{|l|}{ Lamiaceae } \\
\hline Mentha arvensis L. & Wild mint & Cheyenne & stimulant & $\begin{array}{l}\text { leaves } \\
\text { and stem }\end{array}$ & $\begin{array}{l}\text { ground infusion or } \\
\text { boiled to stimulate } \\
\text { vital organs }\end{array}$ & $\mathrm{K}, \mathrm{M}$ \\
\hline
\end{tabular}


Dexter et al. - Prehistoric Plant Use at Beaver Creek Rock Shelter,

\section{Southwestern Montana, U.S.A.}

Table 15. Plants implied from the archaeological record at Beaver Creek Rock Shelter, southwestern Montana, U.S.A. with ethnographic uses as additional medicinal aids. Information sources: (D) Densmore 1927, (G) Grinnell 1972, (H) Hart 1992, (K1) Kindscher 1987, (K2) Kindscher 1992, (M1) Moerman 1998, (M2) Moerman 2003, (S) Smith 1932, and (T) Taylor 1989.

\begin{tabular}{|c|c|c|c|c|c|c|}
\hline \multicolumn{2}{|l|}{ Plant names } & \multirow{2}{*}{$\begin{array}{l}\text { Cultural } \\
\text { source }\end{array}$} & \multicolumn{3}{|c|}{ Plant information } & \multirow[t]{2}{*}{ Sources } \\
\hline Scientific & Common & & Uses & Parts used & Preparation & \\
\hline \multicolumn{7}{|l|}{ Apiaceae } \\
\hline $\begin{array}{l}\text { Angelica dawsonii } \\
\text { S.Watson }\end{array}$ & $\begin{array}{l}\text { Dawson's } \\
\text { angelica }\end{array}$ & Blackfeet & panacea & root & infusion & M1 \\
\hline $\begin{array}{l}\text { Cicuta douglasii } \\
\text { (DC.) J.M.Coult. } \\
\text { \& Rose }\end{array}$ & $\begin{array}{l}\text { Western } \\
\text { water-hemlock }\end{array}$ & MT Indian & snakebite & root & poultice of split root & M1 \\
\hline $\begin{array}{l}\text { Peucedanum } \\
\text { macrocarpum Nutt. }\end{array}$ & $\begin{array}{l}\text { Nineleaf } \\
\text { biscuitroot }\end{array}$ & Blackfeet & panacea & root & $\begin{array}{l}\text { chewed and sprayed } \\
\text { by the mouth to heal } \\
\text { where the root lands }\end{array}$ & M1 \\
\hline $\begin{array}{l}\text { Perideridia montana } \\
\text { (Blank.) Dorn }\end{array}$ & $\begin{array}{l}\text { Gairdner's } \\
\text { yampah }\end{array}$ & Blackfeet & panacea & root & chewed & M1 \\
\hline $\begin{array}{l}\text { Sanicula marilandica } \\
\text { L. }\end{array}$ & $\begin{array}{l}\text { Maryland black } \\
\text { snakeroot }\end{array}$ & Ojibwa & snakebite & root & pounded & M1, S \\
\hline \multicolumn{7}{|l|}{ Brassicaceae } \\
\hline Turritis glabra L. & Tower-mustard & Cheyenne & panacea & unknown & $\begin{array}{l}\text { infusion used to } \\
\text { prevent sickness }\end{array}$ & G, M1 \\
\hline \multicolumn{7}{|l|}{ Fabaceae } \\
\hline Dalea purpurea Vent. & $\begin{array}{l}\text { Purple prairie } \\
\text { clover }\end{array}$ & MT Indian & panacea & root & $\begin{array}{l}\text { pulverized, boiled, } \\
\text { and drunk to } \\
\text { prevent sickness }\end{array}$ & $\mathrm{K} 2, \mathrm{M} 1$ \\
\hline $\begin{array}{l}\text { Psoralea } \\
\text { esculenta Pursh }\end{array}$ & $\begin{array}{l}\text { Pomme- } \\
\text { de-prairie }\end{array}$ & Blackfeet & $\begin{array}{l}\text { orthopedic } \\
\text { aid }\end{array}$ & root & $\begin{array}{l}\text { poultice of chewed } \\
\text { root to heal fractures }\end{array}$ & $\begin{array}{l}\mathrm{H}, \mathrm{K} 1 \\
\mathrm{~K} 2 \\
\mathrm{M} 1, \mathrm{~T}\end{array}$ \\
\hline \multicolumn{7}{|l|}{ Lamiaceae } \\
\hline $\begin{array}{l}\text { Agastache } \\
\text { foeniculum (Pursh) } \\
\text { Kuntze }\end{array}$ & $\begin{array}{l}\text { Lavender } \\
\text { hyssop }\end{array}$ & Cheyenne & diaphoretic & leaves & $\begin{array}{l}\text { steamed to induce } \\
\text { sweating in order } \\
\text { to release toxins }\end{array}$ & $\mathrm{K} 2, \mathrm{M} 1$ \\
\hline \multirow[t]{4}{*}{ Mentha arvensis L. } & \multirow[t]{4}{*}{ Wild mint } & Cree & panacea & unknown & $\begin{array}{l}\text { infusion used to } \\
\text { prevent sickness }\end{array}$ & \begin{tabular}{|l}
$\mathrm{D}, \mathrm{M} 1$ \\
$\mathrm{M} 2$
\end{tabular} \\
\hline & & Blackfeet & panacea & leaves & $\begin{array}{l}\text { beverage to treat a } \\
\text { variety of illnesses }\end{array}$ & $\mathrm{M} 1, \mathrm{M} 2$ \\
\hline & & \multirow[t]{2}{*}{ Ojibwa } & $\begin{array}{l}\text { blood } \\
\text { remedy }\end{array}$ & whole plant & infusion & $\mathrm{M} 1, \mathrm{M} 2$ \\
\hline & & & diaphoretic & unknown & $\begin{array}{l}\text { steamed to induce } \\
\text { sweating in order } \\
\text { to release toxins }\end{array}$ & $\mathrm{M} 1, \mathrm{M} 2$ \\
\hline Monarda fistulosa L. & Beebalm & Salish & panacea & unknown & $\begin{array}{l}\text { infusion to treat } \\
\text { miscellaneous } \\
\text { diseases }\end{array}$ & M1 \\
\hline
\end{tabular}




\section{Summary and Discussion}

Archaeologists have traditionally viewed botanical remains as evidence of prehistoric subsistence, particularly when interpreting macrobotanical remains. This research demonstrates that archaeologists need to use caution in assuming plant remains in the archaeological record are predominately tied to subsistence. Moerman (1996), who summarized the number of Native American food and medicinal plant uses from a large computerized database with 44,775 Native American historic plant uses, demonstrated that medicinal uses were more than twice as numerous as food uses. Of the 44,775 historic uses he recorded $45 \%$ were medicinal, $19 \%$ food, and $29 \%$ both food and medicinal (Moerman 1996:9).

The importance of having medical supplies available is unmistakable. Although we cannot be certain which species of plants are represented in our pollen core, some of the families are heavily laden with species used historically for medicinal purposes. Moerman (1996) calculated the top ten plant families most commonly used for medicinal purposes. Three of the families discovered at the Beaver Creek Rock Shelter are included on Moerman's top ten medicinal list, including Apiaceae (ranked second), Ranunculaceae (ranked fifth), and Lamiaceae (ranked ninth).

Perhaps more noteworthy, as it is unexpected, is that three of the plant families found at the Beaver Creek Rock Shelter were ranked by Moerman among the least-used families for traditional medicinal use, including Brassicaceae (ranked second in the bottom ten), Fabaceae (ranked eighth in the bottom ten), and Cyperaceae (ranked ninth in the bottom ten) (Moerman 1996:5). Finding these three families on Moerman's medicinally least-used list was unexpected as our research showed that in the Northern Plains ethnographies Brassicaceae, Fabaceae, and Cyperaceae families were predominately used for medicinal purposes.

The discrepancy in the research may be due to sample size or geographical considerations. Moerman's research area included North America and our research area encompassed solely Montana and surrounding areas. Furthermore, Moerman's research included 291 tribes, whereas our study was limited to 8 tribes.

The appearance of the 7 plant families-Apiaceae, Brassicaceae, Fabaceae, Geraniaceae, Lamiaceae, Ranunculaceae, and Cyperaceae-in the occupation layers at the Beaver Creek Rock Shelter suggests the plants were utilized by the prehistoric inhabitants of the shelter. Our ethnographic research of Northern Plains traditional uses of plants in these families implies the rock shelter occupants often had medicinal remedies readily available. When an injury or illness strikes, the medicinal plants needed for treatment may not be in season, located in the immedi- ate geographic area, or may require time for preparation, such as drying. Having a prepared supply of treatments could be a major factor in survival. The prehistoric people of the Beaver Creek Rock Shelter appear to have been well adapted to an unpredictable environment, as these same plants reappear in the archaeological record for thousands of years.

\section{Acknowledgments}

The Beaver Creek Rock Shelter research was funded by a Cost Share Agreement between the Helena National Forest and Carroll College. The research would not be possible without support from the Helena National Forest Archaeologists Mark Bodily, Arian Randall, and Kyle McGuire. We also thank anonymous reviewers for their constructive comments.

\section{Literature Cited}

Baugh, T.G. \& J.E. Ericson. 1994. Prehistoric Exchange Systems in North America. Plenum Press, New York, U.S.A.

Blankinship, J.W. 1905. Native Economic Plants of Montana. Montana Agricultural College Experiment Station, Bozeman, Montana, U.S.A.

Boyd, M. 1998. Interdependence and power: Complexity in hunter-gatherer/farmer exchanges. Plains Anthropologist 43(165):311-319. www.jstor.org/stable/25669553

Carlson, R.L. 1994. Trade and exchange in Prehistoric British Columbia. Pp. 307-362 in Prehistoric Exchange Systems in North America. Edited by T.G. Baugh \& J.E. Ericson. Plenum Press, New York, U.S.A.

Clavelle, C.M. 1997. Ethnobotany of Two Cree Communities in the Southern Boreal Forest of Seskatchewan. Unpublished research paper, Department of Anthropology, University of Seskatchewan Saskatoon, Canada.

Cooper, J. 2008. Bison Hunting and Late Prehistoric Human Subsistence Economics in the Great Plains. Ph.D. Dissertation, Department of Anthropology, Southern Methodist University, Dallas, Texas, U.S.A.

Cummings, L.S. \& C. Yost. 2011. Pollen analysis of sediment samples and protein residue analysis of a projectile point from the Beaver Creek Rock Shelter, Montana. Pp. 121-138 in Carroll College 2011 Archeological Field School: Test Excavations at 24LC 1993/2186 Lewis and Clark County, Helena National Forest. Edited by Lauri Travis. Department of Sociology and Anthropology, Carroll College, Helena, Montana, U.S.A. [Submitted to Helena National Forest, Helena, Montana]. 


\section{Dexter et al. - Prehistoric Plant Use at Beaver Creek Rock Shelter, Southwestern Montana, U.S.A.}

Densmore, F. 1927. Use of plants by the Chippewa Indians. Pp. 275-397 in Forty-Fourth Annual Report of the Bureau of American Ethnology to the Secretary of the Smithsonian Institute, 1926-1927. United States Government Printing Office, Washington, U.S.A.

Duke, P. \& M. Wilson. 1994. Cultures of the mountains and plains: From Selkirk Mountains to the Bitterroot Range. Pp. 56-70 in Plains Indians, A.D. 500-1500: The archaeological past of historic groups. Edited by K. Schlesier. University of Oklahoma Press, Norman, Oklahoma, U.S.A.

Dusenberry, V. \& L. Dusenberry Crow. 1998. The Montana Cree: A study in religious persistence. University of Oklahoma Press, Norman, Oklahoma, U.S.A.

Ewers, J.C. 1983. The Blackfeet: Raiders on the Northwestern Plains. University of Oklahoma Press, Norman, Oklahoma, U.S.A.

Faegri, K. \& J. Iverson. 1989. Textbook of Pollen Analysis. Fourth edition. John Wiley and Sons, New York, New York, U.S.A.

Frison, G. 1991. Prehistoric Hunters of the High Plains. Second edition. Academic Press, New York, U.S.A.

Frison, G. 1998. The Northwestern and Northern Plains Archaic. Pp. 140-172 in Archaeology of the Great Plains. Edited by W.R. Wood. University Press of Kansas, Lawrence, Kansas, U.S.A.

Galm, J. 1994. Prehistoric trade and exchange in the Interior Plateau of Northwestern North America. Pp. 275-306 in Prehistoric Exchange Systems in North America. Edited by T.G. Baugh \& J.E. Ericson. Plenum Press, New York, U.S.A.

Gilmore, M.R. 1977. Uses of Plants by Indians of the Missouri River Region. Reprint. University of Nebraska Press, Lincoln, Nebraska, U.S.A.

Greiser, S. 1994. Late Prehistoric cultures on the Montana Plains. Pp. 34-55 in Plains Indians, A.D. 500-1500: The archaeological past of historic groups. Edited by $\mathrm{K}$. Schlesier. University of Oklahoma Press, Norman, Oklahoma, U.S.A.

Grinnell, G.B. 1905. Some Cheyenne plant medicines. American Anthropologists, New Series 7(1):37-43.

Grinnell, G.B. 1972. The Cheyenne Indians, Volume 1: History and society. Bison Books, Lincoln, Nebraska, U.S.A.
Hannus, L.A. 1994. Cultures of the heartland: Beyond the Black Hills. Pp. 176-198 in Plains Indians, A.D. 5001500: The archaeological past of historic groups. Edited by K. Schlesier. University of Oklahoma Press, Norman, Oklahoma, U.S.A.

Hart, J.A. 1981. The ethnobotany of the Northern Cheyenne Indians of Montana. Journal of Ethnopharmacology 4(1):1-55. DOI:10.1016/0378-8741(81)90019-2

Hart, J. 1992. Montana Native Plants and Early Peoples. Montana Historical Society Press, Helena, Montana, U.S.A.

Hoffman, W.J. 1891. The Mide'wiwin or "Grand Medicine Society" of the Ojibwa. Pp. 143-300 in Seventh Annual Report of the Bureau of Ethnology to the Secretary of the Smithsonian Institution, 1885-1886. United States Government Printing Office, Washington, U.S.A.

Holmes, E. 1884. Medicinal plants used by the Cree Indians, Hudson's Bay Territory. American Journal of Pharmacy 56(12):3-7.

Johnston, A. 1970. Blackfoot Indian utilization of the flora of the northwestern great plains. Economic Botany 24(3):301-324. DOI:10.1007/BF02860666.

Johnston, A. 1987. Plants and the Blackfoot. Lethbridge Historical Society Occasional Papers, Historical Society of Alberta Press, Calgary, Alberta, Canada.

Kindscher, K. 1987. Edible Wild Plants of the Prairie: An ethnobotanical guide. University Press of Kansas, Lawrence, Kansas, U.S.A.

Kindscher, K. 1992. Medicinal Wild Plants of the Prairie: An ethnobotanical guide. University Press of Kansas, Lawrence, Kansas, U.S.A.

Lesica, P. 2012. Manual of Montana Vascular Plants. Botanical Research Institute of Texas Press, Fort Worth, Texas, U.S.A.

Lowie, R.H. 1982. Indians of the Plains. Bison Books, Lincoln, Nebraska, U.S.A.

Mincemoyer, S. 2012. Checklist of Montana Vascular Plants: Dated February 14, 2012. Montana Natural Heritage Program, Helena, Montana, U.S.A. http://mtnhp.org/ docs/021412 MT Plant_List.pdf.

Moerman, D. 1996. An analysis of the food plants and drug plants of native North America. Journal of Ethnopharmacology 52(1):1-22. DOI:10.1016/0378-8741(96)01393-1. 
Moerman, D. 1998. Native American Ethnobotany. Timber Press, Portland, Oregon, U.S.A.

Moerman, D. 2003+. Native American Ethnobotany Database. A database of foods, drugs, dyes and fibers of native American peoples, derived from plants. University of Michigan, Dearborn, Michigan, U.S.A. http://herb.umd. umich.edu/

Reaves, R. 2005. Native Plants of Arizona 2005. Electronic document. http://jan.ucc.nau.edu/plants-c/bio414/

Reeves, B. 1970. Cultural Change on the Northwestern Plains, 1000 B.C.-A.D. 1000. Ph.D. Dissertation, University of Calgary, Calgary, Alberta, Canada.

Scheinost, P.L. \& M.E. Stannard. 2010. Sticky Purple Geranium Plant Guide (Geranium viscosissimum). United States Department of Agriculture - Natural Resources Conservation Service, Plant Materials Center, Pullman, Washington, U.S.A.

Schlesier, K. 1994. Plains Indians, A.D. 500-1500: The archaeological past of historic groups. University of Oklahoma Press, Norman, Oklahoma, U.S.A.

Smith, H.H. 1932. Ethnobotany of the Ojibwe Indians. Bulletin of the Public Museum of the City of Milwaukee $4(3): 327-525$.

Taylor, A.R. 1989. Two decades of ethnobotany in the Northwest Plains. International Journal of American Linguistics 55(3):359-381. www.jstor.org/stable/1265075
Teit, J. 1928. The Salishan tribes of the Western Plateaus. Pp. 22-835 in Forty-Fifth Annual Report of the Bureau of American Ethnology to the Secretary of the Smithsonian Institution. Edited by F. Boas. United States Government Printing Office, Washington, U.S.A.

Travis, L. 1988. An archaeological survey in the plains foothills ecotone, Northern Colorado. Plains Anthropologist 33(120):171-186. www.jstor.org/stable/25668754

Travis, L., K. Rausch \& K. Williams. 2011. Carroll College 2011 Archaeological Field School: Test excavations at 24LC1993/2186 Lewis and Clark County, Helena National Forest. Carroll College Department of Sociology and Anthropology, Helena, Montana. Submitted to Helena National Forest, Helena, Montana, U.S.A.

Travis, L., K. Rausch \& K. Williams. 2012. Late Holocene climate change at the Beaver Creek Rock Shelter, Southwestern Montana. Plains Anthropologist 57(223):275289. DOI:10.1179/pan.2012.021

Turner, N.C. \& M.A.M. Bell. 1971. The ethnobotany of the Coast Salish Indians of Vancouver Island. Economic Botany 25(1):63-99. DOI:10.1007/BF02894564

Vehik, S.C. \& T.G. Baugh. 1994. Prehistoric plains trade. Pp. 241-274 in Prehistoric Exchange Systems in North America. Edited by T.G. Baugh \& J.E. Ericson. Plenum Press, New York, U.S.A.

Wood, W.R. 1974. Northern Plains village cultures: Internal stability and external relationships. Journal of Anthropological Research 30(1):1-16. www.jstor.org/stable/3629916 